\title{
Programming and Reasoning with Guarded Recursion for Coinductive Types
}

\author{
Ranald Clouston, Aleš Bizjak, Hans Bugge Grathwohl, and Lars Birkedal \\ Department of Computer Science, Aarhus University, Denmark \\ \{ranald.clouston, abizjak, hbugge, birkedal\}@cs.au.dk
}

\begin{abstract}
We present the guarded lambda-calculus, an extension of the simply typed lambda-calculus with guarded recursive and coinductive types. The use of guarded recursive types ensures the productivity of well-typed programs. Guarded recursive types may be transformed into coinductive types by a type-former inspired by modal logic and Atkey-McBride clock quantification, allowing the typing of acausal functions. We give a call-by-name operational semantics for the calculus, and define adequate denotational semantics in the topos of trees. The adequacy proof entails that the evaluation of a program always terminates. We demonstrate the expressiveness of the calculus by showing the definability of solutions to Rutten's behavioural differential equations. We introduce a program logic with Löb induction for reasoning about the contextual equivalence of programs.
\end{abstract}

\section{Introduction}

The problem of ensuring that functions on coinductive types are well-defined has prompted a wide variety of work into productivity checking, and rule formats for coalgebra. Guarded recursion [10] guarantees productivity and unique solutions by requiring that recursive calls be nested under a constructor, such as cons (written ::) for streams. This can sometimes be established by a simple syntactic check, as for the stream toggle and binary stream function interleave below:

toggle $=1:: 0::$ toggle

interleave $(\mathrm{x}:: \mathrm{xs})$ ys $=\mathrm{x}::$ interleave ys $\mathrm{xs}$

Such syntactic checks, however, are often too blunt and exclude many valid definitions. For example the regular paperfolding sequence, the sequence of left and right turns (encoded as 1 and 0 ) generated by repeatedly folding a piece of paper in half, can be defined via the function interleave as follows [11:

paperfolds $=$ interleave toggle paperfolds

This definition is productive, but the putative definition below, which also applies interleave to two streams and so apparently is just as well-typed, is not:

paperfolds' $=$ interleave paperfolds' toggle 
This equation is satisfied by any stream whose tail is the regular paperfolding sequence, so lacks a unique solution. Unfortunately the syntactic productivity checker of the proof assistant Coq [12] will reject both definitions.

A more flexible approach, first suggested by Nakano [18, is to guarantee productivity via types. A new modality, for which we follow Appel et al. [3] by writing and using the name 'later', allows us to distinguish between data we have access to now, and data which we have only later. This $>$ must be used to guard self-reference in type definitions, so for example guarded streams of natural numbers are defined by the guarded recursive equation

$$
\mathrm{Str}^{\mathrm{g}} \triangleq \mathbf{N} \times \mathrm{Str}^{\mathrm{g}}
$$

asserting that stream heads are available now, but tails only later. The type of interleave will be $\mathrm{Str}^{\mathrm{g}} \rightarrow \mathrm{Str}^{\mathrm{g}} \rightarrow \mathrm{Str}^{\mathrm{g}}$, capturing the fact the (head of the) first argument is needed immediately, but the second argument is needed only later. In term definitions the types of self-references will then be guarded by also. For example interleave paperfolds' toggle becomes ill-formed, as the paperfolds' self-reference has type $\checkmark \mathrm{Str}^{\mathrm{g}}$, rather than $\mathrm{Str}^{\mathrm{g}}$, but interleave toggle paperfolds will be well-formed.

Adding $\longrightarrow$ alone to the simply typed $\lambda$-calculus enforces a discipline more rigid than productivity. For example the obviously productive stream function

$$
\text { every2nd }\left(x:: x^{\prime}:: x s\right)=x:: \text { every } 2 n d x s
$$

cannot be typed because it violates causality [14]: elements of the result stream depend on deeper elements of the argument stream. In some settings, such as reactive programming, this is a desirable property, but for productivity guarantees alone it is too restrictive. We need the ability to remove - in a controlled way. This is provided by the clock quantifiers of Atkey and McBride 4, which assert that all data is available now. This does not trivialise the guardedness requirements because there are side-conditions controlling when clock quantifiers may be introduced. Moreover clock quantifiers transform guarded recursive types into first-class coinductive types, with guarded recursion defining the rule format for their manipulation.

Our presentation departs from Atkey and McBride's [4 by regarding the 'everything now' operator as a unary type-former, written and called 'constant', rather than a quantifier. Observing that the types $A \rightarrow A$ and $A \rightarrow \mathbf{\square} A$ are always inhabited allows us to see the type-former, via the Curry-Howard isomorphism, as an $S_{4}$ modality, and hence base our operational semantics on the established typed calculi for intuitionistic S4 (IS4) of Bierman and de Paiva [5]. This is sufficient to capture all examples in the literature, which use only one clock; for examples that require multiple clocks we suggest extending our calculus to a multimodal logic.

In this paper we present the guarded $\lambda$-calculus, $\mathrm{g} \lambda$, extending the simply typed $\lambda$-calculus with coinductive and guarded recursive types. We define call-by-name operational semantics, which blocks non-termination via recursive definitions 
unfolding indefinitely. We define adequate denotational semantics in the topos of trees [6] and as a consequence prove normalisation. We introduce a program $\operatorname{logic} \operatorname{Lg} \lambda$ for reasoning about the denotations of $\mathrm{g} \lambda$-programs; given adequacy this permits proofs about the operational behaviour of terms. The logic is based on the internal logic of the topos of trees, with modalities $\triangleright, \square$ on predicates, and Löb induction for reasoning about functions on both guarded recursive and coinductive types. We demonstrate the expressiveness of the calculus by showing the definability of solutions to Rutten's behavioural differential equations [20, and show that $\operatorname{Lg} \lambda$ can be used to reason about them, as an alternative to standard bisimulation-based arguments.

We have implemented the $\mathrm{g} \lambda$-calculus in Agda, a process we found helpful when fine-tuning the design of our calculus. The implementation, with many examples, is available at http://cs.au.dk/ hbugge/gl-agda.zip.

\section{Guarded $\lambda$-calculus}

This section presents the guarded $\lambda$-calculus, written $\mathrm{g} \lambda$, its call-by-name operational semantics, and its types, then gives some examples.

Definition 2.1. g $\lambda$-terms are given by the grammar

$$
\begin{aligned}
& t::=x|\langle\rangle| \text { zero }|\operatorname{succ} t|\langle t, t\rangle\left|\pi_{d} t\right| \lambda x . t|t t| \text { fold } t \mid \text { unfold } t \\
& \quad \mid \text { next } t|\operatorname{prev} \sigma . t| \text { box } \sigma . t \mid \text { unbox } t \mid t \circledast t
\end{aligned}
$$

where $d \in\{1,2\}, x$ is a variable and $\sigma=\left[x_{1} \leftarrow t_{1}, \ldots, x_{n} \leftarrow t_{n}\right]$, usually abbreviated $[\vec{x} \leftarrow \vec{t}]$, is a list of variables paired with terms.

$\operatorname{prev}[\vec{x} \leftarrow \vec{t}] . t$ and box $[\vec{x} \leftarrow \vec{t}]$.t bind all variables of $\vec{x}$ in $t$, but not in $\vec{t}$. We write prev $\iota$.t for $\operatorname{prev}[\vec{x} \leftarrow \vec{x}]$.t where $\vec{x}$ is a list of all free variables of $t$. If furthermore $t$ is closed we simply write prev $t$. We will similarly write box $\iota . t$ and boxt. We adopt the convention that prev and box have highest precedence.

We may extend $\mathrm{g} \lambda$ with sums; for space reasons we leave these to App. C]

Definition 2.2. The reduction rules on closed $\mathrm{g} \lambda$-terms are

$$
\begin{array}{rlr}
\pi_{d}\left\langle t_{1}, t_{2}\right\rangle & \mapsto t_{d} & (d \in\{1,2\}) \\
\left(\lambda x . t_{1}\right) t_{2} & \mapsto t_{1}\left[t_{2} / x\right] & \\
\text { unfold fold } t & \mapsto t & \\
\operatorname{prev}[\vec{x} \leftarrow \vec{t}] . t & \mapsto \operatorname{prev} t[\vec{t} / \vec{x}] & (\vec{x} \text { non-empty }) \\
\operatorname{prev} \text { next } t & \mapsto t & \\
\text { unbox (box }[\vec{x} \leftarrow \vec{t}] . t) & \mapsto t[\vec{t} / \vec{x}] \\
\text { next } t_{1} \circledast \text { next } t_{2} & \mapsto \operatorname{next}\left(t_{1} t_{2}\right)
\end{array}
$$

The rules above look like standard $\beta$-reduction, removing 'roundabouts' of introduction then elimination, with the exception of those regarding prev and next. An apparently more conventional $\beta$-rule for these term-formers would be

$$
\operatorname{prev}[\vec{x} \leftarrow \vec{t}] .(\operatorname{next} t) \mapsto t[\vec{t} / \vec{x}]
$$


but where $\vec{x}$ is non-empty this would require us to reduce an open term to derive next $t$. We take the view that reduction of open terms is undesirable within a call-by-name discipline, so first apply the substitution without eliminating prev.

The final rule is not a true $\beta$-rule, as $\circledast$ is neither introduction nor elimination, but is necessary to enable function application under a next and hence allow, for example, manipulation of the tail of a stream. It corresponds to the 'homomorphism' equality for applicative functors [15.

We next impose our call-by-name strategy on these reductions.

Definition 2.3. Values are terms of the form

$$
\langle\rangle \mid \operatorname{succ}^{n} \text { zero }|\langle t, t\rangle| \lambda x . t \mid \text { fold } t \mid \text { box } \sigma . t \mid \text { next } t
$$

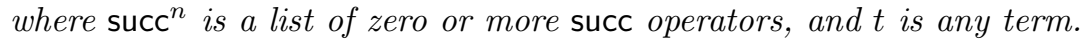

Definition 2.4. Evaluation contexts are defined by the grammar

$$
E::=\cdot|\operatorname{succ} E| \pi_{d} E|E t| \text { unfold } E|\operatorname{prev} E| \operatorname{unbox} E|E \circledast t| v \circledast E
$$

If we regard $\circledast$ as a variant of function application, it is surprising in a call-by-name setting to reduce on both its sides. However both sides must be reduced until they have main connective next before the reduction rule for $\circledast$ may be applied. Thus the order of reductions of $g \lambda$-terms cannot be identified with the call-by-name reductions of the corresponding $\lambda$-calculus term with the novel connectives erased.

Definition 2.5. Call-by-name reduction has format $E[t] \mapsto E[u]$, where $t \mapsto u$ is a reduction rule. From now the symbol $\mapsto$ will be reserved to refer to call-byname reduction. We use $\rightsquigarrow$ for the reflexive transitive closure of $\mapsto$.

Lemma 2.6. The call-by-name reduction relation $\mapsto$ is deterministic.

Definition 2.7. g $\lambda$-types are defined inductively by the rules of Fig. 1 . $\nabla$ is a finite set of type variables. A variable $\alpha$ is guarded in a type $A$ if all occurrences of $\alpha$ are beneath an occurrence of in the syntax tree. We adopt the convention that unary type-formers bind closer than binary type-formers.

$$
\begin{array}{rccc}
\overline{\nabla, \alpha \vdash \alpha} & \frac{\nabla \vdash A_{1} \quad \nabla \vdash A_{2}}{\nabla \vdash \mathbf{1}} \quad \frac{\nabla \vdash A_{1}}{\nabla \vdash \mathbf{N}} & \frac{\nabla \vdash A_{2}}{\nabla \vdash A_{1} \rightarrow A_{2}} \\
\frac{\nabla, \alpha \vdash A}{\nabla \vdash \mu \alpha \cdot A} \alpha \text { guarded in } A & \frac{\nabla \vdash A}{\nabla \vdash \vdash A} & \frac{\cdot \vdash A}{\nabla \vdash \mathbf{\square} A}
\end{array}
$$

Fig. 1. Type formation for the $g \lambda$-calculus 
Note the side condition on the $\mu$ type-former, and the prohibition on $A$ for open $A$, which can also be understood as a prohibition on applying $\mu \alpha$ to any $\alpha$ with above it. The intuition for these restrictions is that unique fixed points exist only where the variable is displaced in time by a $\boldsymbol{\nabla}$, but $\mathbf{\square}$ cancels out this displacement by giving 'everything now'.

Definition 2.8. The typing judgments are given in Fig. 2. There $d \in\{1,2\}$, and the typing contexts $\Gamma$ are finite sets of pairs $x: A$ where $x$ is a variable and A a closed type. Closed types are constant if all occurrences of $\mathbf{a r e}$ beneath an occurrence of $\mathbf{D}$ in their syntax tree.

$$
\begin{aligned}
& \overline{\Gamma, x: A \vdash x: A} \quad \overline{\Gamma \vdash\langle\rangle: \mathbf{1}} \quad \overline{\Gamma \vdash \text { zero }: \mathbf{N}} \quad \frac{\Gamma \vdash t: \mathbf{N}}{\Gamma \vdash \operatorname{succ} t: \mathbf{N}} \\
& \frac{\Gamma \vdash t_{1}: A \quad \Gamma \vdash t_{2}: B}{\Gamma \vdash\left\langle t_{1}, t_{2}\right\rangle: A \times B} \quad \frac{\Gamma \vdash t: A_{1} \times A_{2}}{\Gamma \vdash \pi_{d} t: A_{d}} \quad \frac{\Gamma, x: A \vdash t: B}{\Gamma \vdash \lambda x \cdot t: A \rightarrow B} \\
& \frac{\Gamma \vdash t_{1}: A \rightarrow B \quad \Gamma \vdash t_{2}: A}{\Gamma \vdash t_{1} t_{2}: B} \quad \frac{\Gamma \vdash t: A[\mu \alpha \cdot A / \alpha]}{\Gamma \vdash \text { fold } t: \mu \alpha . A} \quad \frac{\Gamma \vdash t: \mu \alpha \cdot A}{\Gamma \vdash \text { unfold } t: A[\mu \alpha . A / \alpha]} \\
& \begin{array}{cc}
x_{1}: A_{1}, \ldots, x_{n}: A_{n} \vdash t: \bullet A \\
\Gamma \vdash t: A & \Gamma \vdash t_{1}: A_{1} \quad \ldots \quad \Gamma \vdash t_{n}: A_{n} \\
\hline \Gamma \vdash \operatorname{next} t: \triangleright A & \quad A_{1}, \ldots, A_{n} \text { constant }
\end{array} \\
& x_{1}: A_{1}, \ldots, x_{n}: A_{n} \vdash t: A \\
& \frac{\Gamma \vdash t_{1}: A_{1} \quad \cdots \quad C \vdash t_{n}: A_{n}}{\Gamma \vdash \operatorname{box}\left[x_{1} \leftarrow t_{1}, \ldots, x_{n} \leftarrow t_{n}\right] . t: \mathbf{\square} A} A_{1}, \ldots, A_{n} \text { constant } \quad \frac{\Gamma \vdash t: \mathbf{\square} A}{\Gamma \vdash \text { unbox } t: A} \\
& \frac{\Gamma \vdash t_{1}: \triangleright(A \rightarrow B) \quad \Gamma \vdash t_{2}: \triangleright A}{\Gamma \vdash t_{1} \circledast t_{2}: \triangleright B}
\end{aligned}
$$

Fig. 2. Typing rules for the $\mathrm{g} \lambda$-calculus

The constant types exist 'all at once', due to the absence of $\boldsymbol{\sim}$ or presence of $\mathbf{\square}$; this condition corresponds to the freeness of the clock variable in Atkey and McBride 4] (recalling that we use only one clock in this work). Its use as a side-condition to $\mathbf{\square}$-introduction in Fig. 2 recalls (but is more general than) the 'essentially modal' condition for natural deduction for IS4 of Prawitz [19]. The term calculus for IS4 of Bierman and de Paiva [5], on which this calculus is most closely based, uses the still more restrictive requirement that be the main connective. This would preclude some functions that seem desirable, such as the isomorphism $\lambda n$. box $\iota . n: \mathbf{N} \rightarrow \mathbf{\square}$. 
In examples prev usually appears in its syntactic sugar forms

$$
\frac{x_{1}: A_{1}, \ldots, x_{n}: A_{n} \vdash t: \triangleright A}{\Gamma, x_{1}: A_{1}, \ldots, x_{n}: A_{n} \vdash \operatorname{prev} \iota . t: A} A_{1}, \ldots, A_{n} \text { constant } \quad \frac{\vdash t: \triangleright A}{\Gamma \vdash \operatorname{prev} t: A}
$$

and similarly for box; the more general form is nonetheless necessary because (prev $\iota . t)[\vec{u} / \vec{x}]=\operatorname{prev}[\vec{x} \leftarrow \vec{u}] . t$. Getting substitution right in this setting is somewhat delicate. For example our reduction rule $\operatorname{prev}[\vec{x} \leftarrow \vec{t}] . t \mapsto \operatorname{prev} t[\vec{t} / \vec{x}]$ breaches subject reduction on open terms (but not for closed terms). See Bierman and de Paiva [5] for more discussion of substitution with respect to IS4.

Lemma 2.9 (Subject Reduction). $\vdash t: A$ and $t \rightsquigarrow u$ implies $\vdash u: A$.

Example 2.10. (i) The type of guarded recursive streams of natural numbers, $\mathrm{Str}^{\mathrm{g}}$, is defined as $\mu \alpha . \mathbf{N} \times \alpha$. These provide the setting for all examples below, but other definable types include infinite binary trees, as $\mu \alpha$. $\mathbf{N} \times \alpha \times$ $\boldsymbol{\sim} \alpha$, and potentially infinite lists, as $\mu \alpha . \mathbf{1}+(\mathbf{N} \times \boldsymbol{} \times)$.

(ii) We define guarded versions of the standard stream functions cons (written infix as ::), head, and tail as obvious:

$$
\begin{gathered}
:: \triangleq \lambda n . \lambda s . \text { fold }\langle n, s\rangle: \mathbf{N} \rightarrow \triangleright \operatorname{Str}^{\mathrm{g}} \rightarrow \operatorname{Str}^{\mathrm{g}} \\
\mathrm{hd}^{\mathrm{g}} \triangleq \lambda s . \pi_{1} \text { unfold } s: \operatorname{Str}^{\mathrm{g}} \rightarrow \mathbf{N} \quad \mathrm{tt}^{\mathrm{g}} \triangleq \lambda s . \pi_{2} \text { unfold } s:: \mathrm{Str}^{\mathrm{g}} \rightarrow \triangleright \operatorname{Str}^{\mathrm{g}}
\end{gathered}
$$

then use the $\circledast$ term-former for observations deeper into the stream:

$$
\begin{aligned}
& 2 \mathrm{nd}^{\mathrm{g}} \triangleq \lambda s \cdot\left(\text { next hd }{ }^{\mathrm{g}}\right) \circledast\left(\mathrm{tl}^{\mathrm{g}} s\right): \mathrm{Str}^{\mathrm{g}} \rightarrow \mathbf{N} \\
& 3 \mathrm{rd}^{\mathrm{g}} \triangleq \lambda s \cdot\left(\text { next } 2 \mathrm{nd}^{\mathrm{g}}\right) \circledast\left(\mathrm{tt}^{\mathrm{g}} s\right): \mathrm{Str}^{\mathrm{g}} \rightarrow \longrightarrow \mathbf{N} \cdots
\end{aligned}
$$

(iii) Following Abel and Vezzosi [2, Sec. 3.4] we may define a fixed point combinator fix with type $(\rightarrow A \rightarrow A) \rightarrow A$ for any $A$. We use this to define a stream by iteration of a function: iterate takes as arguments a natural number and a function, but the function is not used until the 'next' step of computation, so we may reflect this with our typing:

$$
\text { iterate } \triangleq \lambda f \text {. fix } \lambda g . \lambda n . n::(g \circledast(f \circledast \text { next } n)): \triangleright(\mathbf{N} \rightarrow \mathbf{N}) \rightarrow \mathbf{N} \rightarrow \operatorname{Str}^{g}
$$

We may hence define the guarded stream of natural numbers

$$
\text { nats } \triangleq \text { iterate }(\text { next } \lambda n . \operatorname{succ} n) \text { zero. }
$$

(iv) With interleave, following our discussion in the introduction, we again may reflect in our type that one of our arguments is not required until the next step, defining the term interleave as:

$$
\text { fix } \lambda g . \lambda s . \lambda t .\left(\mathrm{hd}^{\mathrm{g}} s\right)::\left(g \circledast t \circledast \operatorname{next}\left(\mathrm{t}^{\mathrm{g}} s\right)\right): \mathrm{Str}^{\mathrm{g}} \rightarrow \mathrm{Str}^{\mathrm{g}} \rightarrow \mathrm{Str}^{\mathrm{g}}
$$

This typing decision is essential to define the paper folding stream:

$$
\begin{aligned}
\text { toggle } & \triangleq \text { fix } \lambda s .(\text { succ zero })::(\text { next }(\text { zero }:: s)) \\
\text { paperfolds } & \triangleq \text { fix } \lambda s . \text { interleave toggle } s
\end{aligned}
$$


Note that the unproductive definition with interleave $s$ toggle cannot be made to type check: informally, $s: \mathrm{Str}^{\mathrm{g}}$ cannot be converted into a Str ${ }^{\mathrm{g}}$ by prev, as it is in the scope of a variable $s$ whose type Str ${ }^{\mathrm{g}}$ is not constant. To see a less articifial non-example, try to define a filter function on streams which eliminates elements that fail some boolean test.

(v) $\mu$-types are in fact unique fixed points, so carry both final coalgebra and initial algebra structure. To see the latter, observe that we can define

foldr $\triangleq \operatorname{fix} \lambda g \lambda f . \lambda s . f\left\langle\mathrm{hd}^{\mathrm{g}} s, g \circledast\right.$ next $\left.f \circledast \mathrm{tl}^{\mathrm{g}} s\right\rangle:((\mathbf{N} \times \rightarrow A) \rightarrow A) \rightarrow \mathrm{Str}^{\mathrm{g}} \rightarrow A$ and hence for example $\operatorname{map}^{\mathrm{g}} h: \mathrm{Str}^{\mathrm{g}} \rightarrow \operatorname{Str}^{\mathrm{g}}$ is foldr $\lambda x .\left(h \pi_{1} x\right)::\left(\pi_{2} x\right)$.

(vi) The type-former lifts guarded recursive streams to coinductive streams, as we will make precise in Ex. 3.4. Let Str $\triangleq \mathbf{G} \operatorname{tr}^{\mathrm{g}}$. We define hd : Str $\rightarrow \mathbf{N}$ and $\mathrm{tl}: \mathrm{Str} \rightarrow \operatorname{Str}$ by $\mathrm{hd}=\lambda s . \mathrm{hd}^{\mathrm{g}}($ unbox $s)$ and $\mathrm{tl}=\lambda s$. box $\iota \cdot \operatorname{prev} \iota \cdot \mathrm{tl}^{\mathrm{g}}($ unbox $s)$, and hence define observations deep into streams whose results bear no trace of $\boldsymbol{\sim}$, for example $2 \mathrm{nd} \triangleq \lambda s$. hd $(\mathrm{tl} s): \operatorname{Str} \rightarrow \mathbf{N}$.

In general boxed functions lift to functions on boxed types by

$$
\lim \triangleq \lambda f . \lambda x . \text { box } \iota .(\operatorname{unbox} f)(\operatorname{unbox} x): \mathbf{\square}(A \rightarrow B) \rightarrow \mathbf{\square} A \rightarrow \mathbf{\square} B
$$

(vii) The more sophisticated acausal function every2nd : Str $\rightarrow \operatorname{Str}^{\mathrm{g}}$ is

$$
\text { fix } \lambda g . \lambda s .(\text { hd } s)::(g \circledast(\operatorname{next}(\mathrm{tl}(\mathrm{tl} s)))) \text {. }
$$

Note that it must take a coinductive stream Str as argument. The function with coinductive result type is then $\lambda s$. box $\iota$. every2nd $s: \operatorname{Str} \rightarrow \operatorname{Str}$.

\section{Denotational Semantics and Normalisation}

This section gives denotational semantics for $g \lambda$-types and terms, as objects and arrows in the topos of trees [6], the presheaf category over the first infinite ordinal $\omega$ (we give a concrete definition below). These semantics are shown to be sound and, by a logical relations argument, adequate with respect to the operational semantics. Normalisation follows as a corollary of this argument. Note that for space reasons many proofs, and some lemmas, appear in App. A

Definition 3.1. The topos of trees $\mathcal{S}$ has, as objects $X$, families of sets $X_{1}, X_{2}$, ... indexed by the positive integers, equipped with families of restriction functions $r_{i}^{X}: X_{i+1} \rightarrow X_{i}$ indexed similarly. Arrows $f: X \rightarrow Y$ are families of functions $f_{i}: X_{i} \rightarrow Y_{i}$ indexed similarly obeying the naturality condition $f_{i} \circ r_{i}^{X}=r_{i}^{Y} \circ f_{i+1}$.

$\mathcal{S}$ is a cartesian closed category with products defined pointwise. Its exponential $A^{B}$ has, as its component sets $\left(A^{B}\right)_{i}$, the set of $i$-tuples $\left(f_{1}: A_{1} \rightarrow\right.$ $\left.B_{1}, \ldots, f_{i}: A_{i} \rightarrow B_{i}\right)$ obeying the naturality condition, and projections as restriction functions.

Definition 3.2. - The category of sets Set is a full subcategory of $\mathcal{S}$ via the functor $\Delta:$ Set $\rightarrow \mathcal{S}$ with $(\Delta Z)_{i}=Z, r_{i}^{\Delta Z}=i d_{Z}$, and $(\Delta f)_{i}=f$. Objects in this subcategory are called constant objects. In particular the terminal object 1 of $\mathcal{S}$ is $\Delta\{*\}$ and the natural numbers object is $\Delta \mathbb{N}$; 
$-\Delta$ is left adjoint to hom $_{\mathcal{S}}(1,-)$; write $\mathbf{\square}$ for $\Delta \circ \operatorname{hom}_{\mathcal{S}}(1,-): \mathcal{S} \rightarrow \mathcal{S}$. unbox : $\mathbf{a} \rightarrow i d_{\mathcal{S}}$ is the counit of the resulting comonad. Concretely $\operatorname{unbox}_{i}(x)=x_{i}$, i.e. the $i$ 'th component of $x: 1 \rightarrow X$ applied to $*$;

$\rightarrow: \mathcal{S} \rightarrow \mathcal{S}$ is defined by $(-X)_{1}=\{*\}$ and $(-X)_{i+1}=X_{i}$, with $r_{1}^{X}$ defined uniquely and $r_{i+1}^{X}=r_{i}^{X}$. Its action on arrows $f: X \rightarrow Y$ is $(\bullet f)_{1}=i d_{\{*\}}$ and $(-f)_{i+1}=f_{i}$. The natural transformation next $: i d_{\mathcal{S}} \rightarrow \longrightarrow$ has next $_{1}$ unique and next $_{i+1}=r_{i}^{X}$ for any $X$.

Definition 3.3. We interpet types in context $\nabla \vdash A$, where $\nabla$ contains $n$ free variables, as functors $\llbracket \nabla \vdash A \rrbracket:\left(\mathcal{S}^{o p} \times \mathcal{S}\right)^{n} \rightarrow \mathcal{S}$, usually written $\llbracket A \rrbracket$. This mixed variance definition is necessary as variables may appear negatively or positively.

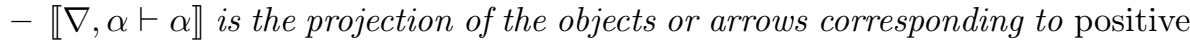
occurrences of $\alpha$, e.g. $\llbracket \alpha \rrbracket(\vec{W}, X, Y)=Y$;

- $\mathbb{1} \rrbracket$ and $\llbracket \mathbf{N} \rrbracket$ are the constant functors $\Delta\{*\}$ and $\Delta \mathbb{N}$ respectively;

- $\llbracket A_{1} \times A_{2} \rrbracket(\vec{W})=\llbracket A_{1} \rrbracket(\vec{W}) \times \llbracket A_{2} \rrbracket(\vec{W})$ and likewise for $\mathcal{S}$-arrows;

- $\llbracket A_{1} \rightarrow A_{2} \rrbracket(\vec{W})=\llbracket A_{2} \rrbracket(\vec{W}) \llbracket A_{2} \rrbracket\left(\vec{W}^{\prime}\right)$ where $\vec{W}^{\prime}$ is $\vec{W}$ with odd and even elements switched to reflect change in polarity, i.e. $\left(X_{1}, Y_{1}, \ldots\right)^{\prime}=\left(Y_{1}, X_{1}, \ldots\right)$;

$-\llbracket \rightarrow A \rrbracket, \llbracket A \rrbracket$ are defined by composition with the functors $\square, \mathbf{\square}$ (Def. 3.2).

$-\llbracket \mu \alpha \cdot A \rrbracket(\vec{W})=\operatorname{Fix}(F)$, where $F:\left(\mathcal{S}^{o p} \times \mathcal{S}\right) \rightarrow \mathcal{S}$ is the functor given by $F(X, Y)=\llbracket A \rrbracket(\vec{W}, X, Y)$ and $\operatorname{Fix}(F)$ is the unique (up to isomorphism) $X$ such that $F(X, X) \cong X$. The existence of such $X$ relies on $F$ being a suitably locally contractive functor, which follows by Birkedal et al [6. Sec. 4.5] and the fact that $\mathbf{\square}$ is only ever applied to closed types. This restriction on $\mathbf{\square}$ necessary because the functor $\mathbf{\square}$ is not strong.

Example 3.4. $\llbracket \mathrm{Str}^{\mathrm{g}} \rrbracket_{i}=\mathbb{N}^{i}$, with projections as restriction functions, so is an object of approximations of streams - first the head, then the first two elements, and so forth. $\llbracket \operatorname{Str} \rrbracket_{i}=\mathbb{N}^{\omega}$ at all levels, so is the constant object of streams. More generally, any polynomial functor $F$ on Set can be assigned a $g \lambda$-type $A_{F}$ with a free type variable $\alpha$ that occurs guarded. The denotation of $\boldsymbol{\square} \mu \alpha \cdot A_{F}$ is the constant object of the carrier of the final coalgebra for $F$ [17, Thm. 2].

Lemma 3.5. The interpretation of a recursive type is isomorphic to the interpretation of its unfolding: $\llbracket \mu \alpha \cdot A \rrbracket(\vec{W}) \cong \llbracket A[\mu \alpha \cdot A / \alpha] \rrbracket(\vec{W})$.

Lemma 3.6. Closed constant types denote constant objects in $\mathcal{S}$.

Note that the converse does not apply; for example $\llbracket 1 \rrbracket$ is a constant object.

Definition 3.7. We interpret typing contexts $\Gamma=x_{1}: A_{1}, \ldots, x_{n}: A_{n}$ as $\mathcal{S}$-objects $\llbracket \Gamma \rrbracket \triangleq \llbracket A_{1} \rrbracket \times \cdots \times \llbracket A_{n} \rrbracket$ and hence interpret typed terms-in-context $\Gamma \vdash t: A$ as $\mathcal{S}$-arrows $\llbracket \Gamma \vdash t: A \rrbracket: \llbracket \Gamma \rrbracket \rightarrow \llbracket A \rrbracket$ (usually written $\llbracket t \rrbracket$ ) as follows.

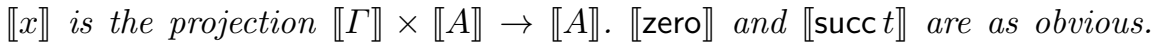
Term-formers for products and function spaces are interpreted via the cartesian closed structure of $\mathcal{S}$. Exponentials are not pointwise, so we give explicitly: 
- $\llbracket \lambda x . t \rrbracket_{i}(\gamma)_{j}$ maps $a \mapsto \llbracket \Gamma, x: A \vdash t: B \rrbracket_{j}\left(\gamma \uparrow_{j}, a\right)$, where $\gamma \uparrow_{j}$ is the result of applying restriction functions to $\gamma \in \llbracket \Gamma \rrbracket_{i}$ to get an element of $\llbracket \Gamma \rrbracket_{j}$;

$-\llbracket t_{1} t_{2} \rrbracket_{i}(\gamma)=\left(\llbracket t_{1} \rrbracket_{i}(\gamma)_{i}\right) \circ \llbracket t_{2} \rrbracket_{i}(\gamma) ;$

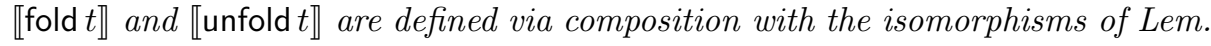

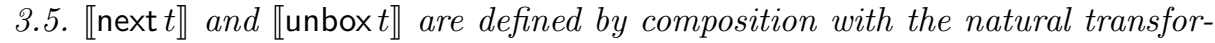
mations introduced in Def. 3.2. The final three cases are

$-\llbracket \operatorname{prev}\left[x_{1} \leftarrow t_{1}, \ldots . . t \rrbracket_{i}(\gamma) \triangleq \llbracket t \rrbracket_{i+1}\left(\llbracket t_{1} \rrbracket_{i}(\gamma), \ldots\right)\right.$, where $\llbracket t_{1} \rrbracket_{i}(\gamma) \in \llbracket A_{1} \rrbracket_{i}$ is also in $\llbracket A_{1} \rrbracket_{i+1}$ by Lem. 3.6;

- $\llbracket$ box $\left[x_{1} \leftarrow t_{1}, \ldots\right] . t \rrbracket_{i}(\gamma)_{j}=\llbracket t \rrbracket_{j}\left(\llbracket t_{1} \rrbracket_{i}(\gamma), \ldots\right)$, again using Lem. 3.6;

- $\llbracket t_{1} \circledast t_{2} \rrbracket_{1}$ is defined uniquely; $\llbracket t_{1} \circledast t_{2} \rrbracket_{i+1}(\gamma) \triangleq\left(\llbracket t_{1} \rrbracket_{i+1}(\gamma)_{i}\right) \circ \llbracket t_{2} \rrbracket_{i+1}(\gamma)$.

Lemma 3.8. Given typed terms in context $x_{1}: A_{1}, \ldots, x_{m}: A_{m} \vdash t: A$ and $\Gamma \vdash t_{k}: A_{k}$ for $1 \leq k \leq m, \llbracket t[\vec{t} / \vec{x}] \rrbracket_{i}(\gamma)=\llbracket t \rrbracket_{i}\left(\llbracket t_{1} \rrbracket_{i}(\gamma), \ldots, \llbracket t_{m} \rrbracket_{i}(\gamma)\right)$.

Theorem 3.9 (Soundness). If $t \rightsquigarrow u$ then $\llbracket t \rrbracket=\llbracket u \rrbracket$.

We now define a logical relation between our denotational semantics and terms, from which both normalisation and adequacy will follow. Doing this inductively proves rather delicate, because induction on size will not support reasoning about our values, as fold refers to a larger type in its premise. This motivates a notion of unguarded size under which $A[\mu \alpha . A / \alpha]$ is 'smaller' than $\mu \alpha$.A. But under this metric $A$ is smaller than $A$, so next now poses a problem. But the meaning of $A$ at index $i+1$ is determined by $A$ at index $i$, and so, as in Birkedal et al [7, our relation will also induct on index. This in turn creates problems with box, whose meaning refers to all indexes simultaneously, motivating a notion of box depth, allowing us finally to attain well-defined induction.

Definition 3.10. The unguarded size us of an open type follows the obvious definition for type size, except that us $(A)=0$.

The box depth bd of an open type is

$-\operatorname{bd}(A)=0$ for $A \in\{\alpha, \mathbf{0}, \mathbf{1}, \mathbf{N}\}$;

- $\mathrm{bd}(A \times B)=\min (\mathrm{bd}(A), \mathrm{bd}(B))$, and similarly for $\mathrm{bd}(A \rightarrow B)$;

$-\mathrm{bd}(\mu \alpha . A)=\mathrm{bd}(A)$, and similarly for $\mathrm{bd}(-A)$;

$-\operatorname{bd}(\square)=\operatorname{bd}(A)+1$.

Lemma 3.11. (i) a guarded in $A$ implies us $(A[B / \alpha]) \leq \mathrm{us}(A)$.

(ii) $\mathrm{bd}(B) \leq \mathrm{bd}(A)$ implies $\mathrm{bd}(A[B / \alpha]) \leq \mathrm{bd}(A)$

Definition 3.12. The family of relations $R_{i}^{A}$, indexed by closed types $A$ and positive integers $i$, relates elements of the semantics $a \in \llbracket A \rrbracket_{i}$ and closed typed terms $t: A$ and is defined as

$-* R_{i}^{1} t$ iff $t \rightsquigarrow\langle\rangle$;

$-n R_{i}^{\mathbf{N}} t$ iff $t \rightsquigarrow \operatorname{succ}^{n}$ zero;

- $\left(a_{1}, a_{2}\right) R_{i}^{A_{1} \times A_{2}} t$ iff $t \rightsquigarrow\left\langle t_{1}, t_{2}\right\rangle$ and $a_{d} R_{i}^{A_{d}} t_{d}$ for $d \in\{1,2\}$; 
- $f R_{i}^{A \rightarrow B} t$ iff $t \rightsquigarrow \lambda$ x.s and for all $j \leq i, a R_{j}^{A} u$ implies $f_{j}(a) R_{j}^{B} s[u / x]$;

$-a R_{i}^{\mu \alpha . A} t$ iff $t \rightsquigarrow$ fold $u$ and $h_{i}(a) R_{i}^{A[\mu \alpha \cdot A / \alpha]} u$, where $h$ is the "unfold" isomorphism for the recursive type (ref. Lem. 3.5);

- $a R_{i}^{A} t$ iff $t \rightsquigarrow$ next $u$ and, where $i>1, a R_{i-1}^{A} u$.

- $a R_{i}^{A} t$ iff $t \rightsquigarrow$ box $u$ and for all $j, a_{j} R_{j}^{A} u$;

This is well-defined by induction on the lexicographic ordering on box depth, then index, then unguarded size. First the case strictly decreases box depth, and no other case increases it (ref. Lem. 3.11. (ii) for $\mu$-types). Second the case strictly decreases index, and no other case increases it (disregarding $\mathbf{\square}$ ). Finally all other cases strictly decrease unguarded size, as seen via Lem. 3.11.(i) for $\mu$-types.

Lemma 3.13 (Fundamental Lemma). Take $\Gamma=\left(x_{1}: A_{1}, \ldots, x_{m}: A_{m}\right)$, $\Gamma \vdash t: A$, and $\vdash t_{k}: A_{k}$ for $1 \leq k \leq m$. Then for all $i$, if $a_{k} R_{i}^{A_{k}} t_{k}$ for all $k$, then

$$
\llbracket \Gamma \vdash t: A \rrbracket_{i}(\vec{a}) R_{i}^{A} t[\vec{t} / \vec{x}] .
$$

\section{Theorem 3.14 (Adequacy and Normalisation).}

(i) For all closed terms $\vdash t: A$ it holds that $\llbracket t \rrbracket_{i} R_{i}^{A} t$;

(ii) $\llbracket \vdash t: \mathbf{N} \rrbracket_{i}=n$ implies $t \rightsquigarrow \operatorname{succ}^{n}$ zero;

(iii) All closed typed terms evaluate to a value.

Proof. (i) specialises Lem. 3.13 to closed types. (ii), (iii) hold by $(i)$ and inspection of Def. 3.12,

Definition 3.15. Typed contexts with typed holes are defined as obvious. Two terms $\Gamma \vdash t: A, \Gamma \vdash u: A$ are contextually equivalent, written $t \simeq_{\mathrm{ctx}} u$, if for all closing contexts $C$ of type $\mathbf{N}$, the terms $C[t]$ and $C[u]$ reduce to the same value.

Corollary 3.16. $\llbracket t \rrbracket=\llbracket u \rrbracket$ implies $t \simeq_{\mathrm{ct} \times} u$.

Proof. $\llbracket C[t] \rrbracket=\llbracket C[u] \rrbracket$ by compositionality of the denotational semantics . Then by Thm. 3.14((ii) they reduce to the same value.

\section{Logic for Guarded Lambda Calculus}

This section presents our program logic $L g \lambda$ for the guarded $\lambda$-calculus. The logic is an extension of the internal language of $\mathcal{S}$ [6]9]. Thus it extends multisorted intuitionistic higher-order logic with two propositional modalities $\triangleright$ and $\square$, pronounced later and always respectively. The term language of $\operatorname{Lg} \lambda$ includes the terms of $\mathrm{g} \lambda$, and the types of $\operatorname{Lg} \lambda$ include types definable in $\mathrm{g} \lambda$. We write $\Omega$ for the type of propositions, and also for the subobject classifier of $\mathcal{S}$.

The rules for definitional equality extend the usual $\beta \eta$-laws for functions and products with new equations for the new $g \lambda$ constructs, listed in Fig. 3 ,

Definition 4.1. A type $X$ is total and inhabited if the formula Total $(X) \equiv$ $\forall x: \triangleright X, \exists x^{\prime}: X, \operatorname{next}\left(x^{\prime}\right)=\triangleright X x$ is valid. 


$$
\begin{aligned}
& \frac{\Gamma \vdash t: A[\mu \alpha \cdot A / \alpha]}{\Gamma \vdash \operatorname{unfold}(\text { fold } t)=t} \quad \frac{\Gamma \vdash t: \mu \alpha . A}{\Gamma \vdash \text { fold }(\text { unfold } t)=t} \quad \frac{\Gamma \vdash t_{1}: A \rightarrow B}{\Gamma \vdash \operatorname{next} t_{1} \circledast \operatorname{next} t_{2}=\operatorname{next}\left(t_{1} t_{2}\right)} \\
& \frac{\Gamma_{\mathbf{\square}} \vdash t: A \quad \Gamma \vdash \vec{t}: \Gamma_{\mathbf{\square}}}{\Gamma \vdash \operatorname{prev}[\vec{x} \leftarrow \vec{t}] .(\operatorname{next} t)=t[\vec{t} / \vec{x}]} \quad \frac{\Gamma_{\mathbf{\square}} \vdash t: A^{\prime} \quad \Gamma \vdash \vec{t}: \Gamma_{\mathbf{\square}}}{\Gamma \vdash \operatorname{next}(\operatorname{prev}[\vec{x} \leftarrow \vec{t}] . t)=t[\vec{t} / \vec{x}]} \\
& \frac{\Gamma \cdot t: A \quad \Gamma \vdash \vec{t}: \Gamma \mathbf{\square}}{\Gamma \vdash \operatorname{unbox}(\operatorname{box}[\vec{x} \leftarrow \vec{t}] . t)=t[\vec{t} / \vec{x}]} \quad \frac{\Gamma \cdot t: \mathbf{\square} A \quad \Gamma \vdash \vec{t}: \Gamma \mathbf{\square}}{\Gamma \vdash \operatorname{box}[\vec{x} \leftarrow \vec{t}] . \text { unbox } t=t[\vec{t} / \vec{x}]}
\end{aligned}
$$

Fig. 3. Additional equations. The context $\Gamma_{\mathbf{m}}$ is assumed constant.

All of the $\mathrm{g} \lambda$-types defined in Sec. 2 are total and inhabited (see App. E for a proof using the semantics of the logic), but that is not the case when we include sum types as the empty type is not inhabited.

Corresponding to the modalities $\boldsymbol{D}$ and $\mathbf{\square}$ on types, we have modalities $\triangleright$ and $\square$ on formulas. The modality $\triangleright$ is used to express that a formula holds only "later", that is, after a time step. It is given by a function symbol $\triangleright: \Omega \rightarrow \Omega$. The $\square$ modality is used to express that a formula holds for all time steps. Unlike the $\triangleright$ modality, $\square$ on formulas does not arise from a function on $\Omega[8$. As with box, it is only well-behaved in constant contexts, so we will only allow $\square$ in such contexts. The rules for $\triangleright$ and $\square$ are listed in Fig. 4 .

$$
\begin{aligned}
& \overline{\Gamma \mid \Xi,(\triangleright \phi \Rightarrow \phi) \vdash \phi} \text { Lӧв } \overline{\Gamma, x: X \mid \exists y: Y, \triangleright \phi(x, y) \vdash \triangleright(\exists y: Y, \phi(x, y))} \exists \triangleright \\
& \overline{\Gamma, x: X \mid \triangleright(\forall y: Y, \phi(x, y)) \vdash \forall y: Y, \triangleright \phi(x, y)} \forall \triangleright \quad \overline{\Gamma \mid \Xi, \phi \vdash \triangleright \phi} \\
& \frac{\star \in\{\wedge, \vee, \Rightarrow\}}{\Gamma \mid \triangleright(\phi \star \psi) \dashv \triangleright \phi \star \triangleright \psi} \quad \frac{\Gamma \mid \neg \neg \phi \vdash \psi}{\Gamma \mid \phi \vdash \square \psi} \quad \frac{\Gamma \mid \phi \vdash \square \psi}{\Gamma \mid \neg \neg \phi \vdash \psi} \quad \frac{\Gamma \mid \phi \vdash \psi}{\Gamma \mid \square \phi \vdash \square \psi} \\
& \overline{\Gamma \mid \square \phi \vdash \phi} \quad \overline{\Gamma \mid \square \phi \vdash \square \square \phi} \quad \overline{\forall x, y: X . \triangleright(x=X y) \Leftrightarrow \operatorname{next} x=\triangleright X \operatorname{next} y} \mathrm{EQ}_{\text {next }}^{\triangleright}
\end{aligned}
$$

Fig. 4. Rules for $\triangleright$ and $\square$. The judgement $\Gamma \mid \Xi \vdash \phi$ expresses that in typing context $\Gamma$, hypotheses in $\Xi$ prove $\phi$. The converse entailment in $\forall \triangleright$ and $\exists \triangleright$ rules holds if $Y$ is total and inhabited. In all rules involving the $\square$ the context $\Gamma$ is assumed constant.

The $\triangleright$ modality can in fact be defined in terms of lift $: \Omega \rightarrow \Omega$ (called succ by Birkedal et al [6]) as $\triangleright=$ lift o next. The lift function will be useful since it allows us to define predicates over guarded types, such as predicates on $\mathrm{Str}^{\mathrm{g}}$.

The semantics of the logic is given in $\mathcal{S}$; terms are interpreted as morphisms of $\mathcal{S}$ and formulas are interpreted via the subobject classifier. We do not present 
the semantics here; except for the new terms of $g \lambda$, whose semantics are defined in Sec. 3, the semantics are as in 6 6 .

Later we will come to the problem of proving $x=\mathbf{m}_{A} y$ from unbox $x={ }_{A}$ unbox $y$, where $x, y$ have type $A$. This in general does not hold, but using the semantics of $\operatorname{Lg} \lambda$ we can prove the proposition below.

Proposition 4.2. The formula $\square\left(\operatorname{unbox} x={ }_{A}\right.$ unbox $\left.y\right) \Rightarrow x=\mathbf{\square}_{A} y$ is valid.

There exists a fixed-point combinator of type $(\rightarrow A \rightarrow A) \rightarrow A$ for all types $A$ in the logic (not only those of in $g \lambda$ ) [6, Thm. 2.4]; we also write fix for it.

Proposition 4.3. For any term $f: \rightarrow A \rightarrow A$ we have fix $f={ }_{A} f(\operatorname{next}(\operatorname{fix} f))$ and, if $u$ is any other term such that $f(\operatorname{next} u)={ }_{A} u$, then $u={ }_{A}$ fix $f$.

In particular this can be used for recursive definitions of predicates. For instance if $P: \mathbf{N} \rightarrow \Omega$ is a predicate on natural numbers we can define a predicate $P_{\text {Str }}$ on $\mathrm{Str}^{\mathrm{g}}$ expressing that $P$ holds for all elements of the stream:

$$
P_{\mathrm{Str}^{\mathrm{g}}} \triangleq \mathrm{fix} \lambda r \cdot \lambda x s . P\left(\mathrm{hd}^{\mathrm{g}} x s\right) \wedge \operatorname{lift}\left(r \circledast\left(\mathrm{tl}^{\mathrm{g}} x s\right)\right): \mathrm{Str}^{\mathrm{g}} \rightarrow \Omega .
$$

The logic may be used to prove contextual equivalence of programs:

Theorem 4.4. Let $t_{1}$ and $t_{2}$ be two $\mathrm{g} \lambda$ terms of type $A$ in context $\Gamma$. If the sequent $\Gamma \mid \emptyset \vdash t_{1}={ }_{A} t_{2}$ is provable then $t_{1}$ and $t_{2}$ are contextually equivalent.

Proof. Recall that equality in the internal logic of a topos is just equality of morphisms. Hence $t_{1}$ and $t_{2}$ denote same morphism from $\Gamma$ to $A$. Adequacy (Cor. 3.16) then implies that $t_{1}$ and $t_{2}$ are contextually equivalent.

Example 4.5. We list some properties provable using the logic. Except for the first property all proof details are in App. B.

(i) For any $f: A \rightarrow B$ and $g: B \rightarrow C$ we have

$$
\left(\operatorname{map}^{\mathrm{g}} f\right) \circ\left(\operatorname{map}^{\mathrm{g}} g\right)=\operatorname{Str}^{\mathrm{g}} \rightarrow \operatorname{Str}^{\mathrm{g}} \operatorname{map}^{\mathrm{g}}(f \circ g) .
$$

Unfolding the definition of mapg from Ex. 2.10(vi) and using $\beta$-rules and Prop. 4.3 we have map $f x s=f\left(\mathrm{hd}^{\mathrm{g}} x s\right)::\left(\operatorname{next}\left(\operatorname{map}^{\mathrm{g}} f\right) \circledast\left(\mathrm{t}^{\mathrm{g}} x s\right)\right)$. Equality of functions is extensional so we have to prove

$$
\Phi \triangleq \forall x s: \operatorname{Str}^{\mathrm{g}}, \operatorname{map}^{\mathrm{g}} f\left(\operatorname{map}^{\mathrm{g}} g x s\right)=\operatorname{Str}^{\mathrm{g}} \operatorname{map}^{\mathrm{g}}(f \circ g) x s .
$$

The proof is by Löb induction, so we assume $\triangleright \Phi$ and take $x s: \operatorname{Str}^{\mathrm{g}}$. Using the above property of map ${ }^{\mathrm{g}}$ we unfold $\operatorname{map}^{\mathrm{g}} f\left(\operatorname{map}^{\mathrm{g}} g x s\right)$ to

$$
f\left(g\left(\mathrm{hd}^{\mathrm{g}} x s\right)\right)::\left(\operatorname{next}\left(\operatorname{map}^{\mathrm{g}} f\right) \circledast\left(\left(\operatorname{next}\left(\operatorname{map}^{\mathrm{g}} g\right)\right) \circledast \mathrm{tl}^{\mathrm{g}} x s\right)\right)
$$

and we unfold map ${ }^{\mathrm{g}}(f \circ g) x s$ to $f\left(g\left(\mathrm{hd}^{\mathrm{g}} x s\right)\right)::\left(\operatorname{next}\left(\operatorname{map}^{\mathrm{g}}(f \circ g)\right) \circledast \mathrm{tl}^{\mathrm{g}} x s\right)$. Since $\mathrm{Str}^{\mathrm{g}}$ is a total type there is a $x s^{\prime}: \mathrm{Str}^{\mathrm{g}}$ such that next $x s^{\prime}=\mathrm{tl}^{\mathrm{g}} x s$. Using this and the rule for $\circledast$ we have

$$
\operatorname{next}\left(\operatorname{map}^{\mathrm{g}} f\right) \circledast\left(\left(\operatorname{next}\left(\operatorname{map}^{\mathrm{g}} g\right)\right) \circledast \mathrm{tl}^{\mathrm{g}} x s\right)=\boldsymbol{S t r}^{\mathrm{g}} \operatorname{next}\left(\operatorname{map}^{\mathrm{g}} f\left(\operatorname{map}^{\mathrm{g}} g x s^{\prime}\right)\right)
$$

and $\operatorname{next}\left(\operatorname{map}^{\mathrm{g}}(f \circ g)\right) \circledast \mathrm{tl}^{\mathrm{g}} x s=\mathrm{Str}^{\mathrm{g}} \operatorname{next}\left(\operatorname{map}^{\mathrm{g}}(f \circ g) x s^{\prime}\right)$. From the induction hypothesis $\triangleright \Phi$ we have $\triangleright\left(\operatorname{map}^{\mathrm{g}}(f \circ g) x s^{\prime}=\operatorname{Str}^{\mathrm{g}} \operatorname{map}^{\mathrm{g}} f\left(\operatorname{map}^{\mathrm{g}} g x s^{\prime}\right)\right)$ and so rule $\mathrm{EQ}_{\text {next }}^{\triangleright}$ concludes the proof. 
(ii) We can also reason about acausal functions. For any $n: \mathbf{N}, f: \mathbf{N} \rightarrow \mathbf{N}$,

$$
\text { every } 2 \text { nd }(\text { box } \iota \text {. iterate }(\text { next } f) n)=\mathrm{Str}_{\text {g }} \text { iterate }\left(\text { next } f^{2}\right) n \text {, }
$$

where $f^{2}$ is $\lambda m . f(f m)$. The proof again uses Löb induction.

(iii) Since our logic is higher-order we can state and prove very general properties, for instance the following general property of map

$$
\begin{aligned}
& \forall P, Q:(\mathbf{N} \rightarrow \Omega), \forall f: \mathbf{N} \rightarrow \mathbf{N},(\forall x: \mathbf{N}, P(x) \Rightarrow Q(f(x))) \\
& \Rightarrow \forall x s: \operatorname{Str}^{\mathrm{g}}, P_{\mathrm{Str}}(x s) \Rightarrow Q_{\mathrm{Str}}\left(\operatorname{map}^{\mathrm{g}} f x s\right) .
\end{aligned}
$$

The proof illustrates the use of the property lift $\circ$ next $=\triangleright$.

(iv) Given a closed term (we can generalise to terms in constant contexts) $f$ of type $A \rightarrow B$ we have box $f$ of type $\mathbf{\square}(A \rightarrow B)$. Define $\mathcal{L}(f)=\lim ($ box $f)$ of type $A \rightarrow \boldsymbol{\square}$. For any closed term $f: A \rightarrow B$ and $x: \boldsymbol{\square}$ we can then prove $\operatorname{unbox}(\mathcal{L}(f) x)={ }_{B} f(\operatorname{unbox} x)$. Then using Prop. 4.2 we can, for instance, prove $\mathcal{L}(f \circ g)=\mathcal{L}(f) \circ \mathcal{L}(g)$.

For functions of arity $k$ we define $\mathcal{L}_{k}$ using $\mathcal{L}$, and analogous properties hold, e.g. we have $\operatorname{unbox}\left(\mathcal{L}_{2}(f) x y\right)=f(\operatorname{unbox} x)($ unbox $y)$, which allows us to transfer equalities proved for functions on guarded types to functions on

口'd types; see Sec. [5] for an example.

\section{Behavioural Differential Equations in $g \lambda$}

In this section we demonstrate the expressivity of our approach by showing how to construct solutions to behavioural differential equations [20] in $\mathrm{g} \lambda$, and how to reason about such functions in $\operatorname{Lg} \lambda$, rather than with bisimulation as is more traditional. These ideas are best explained via a simple example.

Supposing addition $+: \mathbf{N} \rightarrow \mathbf{N} \rightarrow \mathbf{N}$ is given, then pointwise addition of streams, plus, can be defined by the following behavioural differential equation

$$
\operatorname{hd}\left(\text { plus } \sigma_{1} \sigma_{2}\right)=\operatorname{hd} \sigma_{1}+\text { hd } \sigma_{2} \quad \operatorname{tl}\left(\text { plus } \sigma_{1} \sigma_{2}\right)=\operatorname{plus}\left(\mathrm{tl} \sigma_{1}\right)\left(\mathrm{tl} \sigma_{2}\right) \text {. }
$$

To define the solution to this behavioural differential equation in $\mathrm{g} \lambda$, we first translate it to a function on guarded streams plus ${ }^{\mathrm{g}}: \mathrm{Str}^{\mathrm{g}} \rightarrow \mathrm{Str}^{\mathrm{g}} \rightarrow \mathrm{Str}^{\mathrm{g}}$, as

$$
\text { plus }^{\mathrm{g}} \triangleq \operatorname{fix} \lambda f \cdot \lambda s_{1} \cdot \lambda s_{2} \cdot\left(\mathrm{hd}^{\mathrm{g}} s_{1}+\mathrm{hd}^{\mathrm{g}} s_{2}\right)::\left(f \circledast\left(\mathrm{tl}^{\mathrm{g}} s_{1}\right) \circledast\left(\mathrm{tl}^{\mathrm{g}} s_{2}\right)\right)
$$

then define plus : Str $\rightarrow$ Str $\rightarrow$ Str by plus $=\mathcal{L}_{2}\left(\right.$ plus $\left.^{\mathrm{g}}\right)$. By Prop. 4.3 we have

$$
\text { plus }^{\mathrm{g}}=\lambda s_{1} \cdot \lambda s_{2} \cdot\left(\mathrm{hd}^{\mathrm{g}} s_{1}+\mathrm{hd}^{\mathrm{g}} s_{2}\right)::\left(\left(\text { next plus }{ }^{\mathrm{g}}\right) \circledast\left(\mathrm{t}^{\mathrm{g}} s_{1}\right) \circledast\left(\mathrm{tl}^{\mathrm{g}} s_{2}\right)\right) .
$$

This definition of plus satisfies the specification given by the behavioural differential equation above. Let $\sigma_{1}, \sigma_{2}$ : Str and recall that $\mathrm{hd}=\mathrm{hd}^{\mathrm{g}} \circ \lambda s$. unbox $s$. Then use Ex. 4.5.(iv) and equality (1) to get hd $\left(\right.$ plus $\left.\sigma_{1} \sigma_{2}\right)=$ hd $\sigma_{1}+$ hd $\sigma_{2}$.

For $\mathrm{tl}$ we proceed similarly, also using that $\mathrm{t}^{\mathrm{g}}(\operatorname{unbox} \sigma)=\operatorname{next}(\operatorname{unbox}(\mathrm{tl} \sigma))$ which can be proved using the $\beta$-rule for box and the $\eta$-rule for next. 
Since plus $^{\mathrm{g}}$ is defined via guarded recursion we can reason about it with Löb induction, for example to prove that it is commutative. Ex. 4.5.(iv) and Prop. 4.2 then immediately give that plus on coinductive streams Str is commutative.

Once we have defined plus $^{\mathrm{g}}$ we can use it when defining other functions on streams, for instance stream multiplication $\otimes$ which is specified by equations

$\operatorname{hd}\left(\sigma_{1} \otimes \sigma_{2}\right)=\left(\right.$ hd $\left.\sigma_{1}\right) \cdot\left(\right.$ hd $\left.\sigma_{2}\right) \quad \operatorname{tl}\left(\sigma_{1} \otimes \sigma_{2}\right)=\left(\rho\left(\right.\right.$ hd $\left.\left.\sigma_{1}\right) \otimes\left(\mathrm{tl} \sigma_{2}\right)\right) \oplus\left(\left(\mathrm{tl} \sigma_{1}\right) \otimes \sigma_{2}\right)$

where $\rho(n)$ is a stream with head $n$ and tail a stream of zeros, and · is multiplication of natural numbers, and using $\oplus$ as infix notation for plus. We can define $\otimes^{\mathrm{g}}: \mathrm{Str}^{\mathrm{g}} \rightarrow \mathrm{Str}^{\mathrm{g}} \rightarrow \mathrm{Str}^{\mathrm{g}}$ by $\otimes^{\mathrm{g}} \triangleq$

$$
\begin{aligned}
\text { fix } \lambda f \cdot \lambda s_{1} \cdot \lambda s_{2} \cdot\left(\left(\mathrm{hd}^{\mathrm{g}} s_{1}\right) \cdot\left(\mathrm{hd}^{\mathrm{g}} s_{2}\right)\right):: \\
\quad(\text { next plus } \\
\end{aligned}
$$

then define $\otimes=\mathcal{L}_{2}\left(\otimes^{\mathrm{g}}\right)$. It can be shown that the function $\otimes$ so defined satisfies the two defining equations above. Note that the guarded plus ${ }^{\mathrm{g}}$ is used to define $\otimes^{\mathrm{g}}$, so our approach is modular in the sense of [16].

The example above generalises, as we can show that any solution to a behavioural differential equation in Set can be obtained via guarded recursion together with $\mathcal{L}_{k}$. The formal statement is somewhat technical and can be found in App. D.

\section{Discussion}

Following Nakano [18, the modality has been used as type-former for a number of $\lambda$-calculi for guarded recursion. Nakano's calculus and some successors [14212] permit only causal functions. The closest such work to ours is that of Abel and Vezzosi 2, but due to a lack of destructor for their (strong) normalisation result relies on a somewhat artificial operational semantics where the number of nexts that can be reduced under is bounded by some fixed natural number.

Atkey and McBride's extension of such calculi to acausal functions [4 forms the basis of this paper. We build on their work by (aside from various minor changes such as eliminating the need to work modulo first-class type isomorphisms) introducing normalising operational semantics, an adequacy proof with respect to the topos of trees, and a program logic.

An alterative approach to type-based productivity guarantees are sized types, introduced by Hughes et al [13] and now extensively developed, for example integrated into a variant of System $F_{\omega}[1]$. Our approach offers some advantages, such as adequate denotational semantics, and a notion of program proof without appeal to dependent types, but extensions with realistic language features (e.g. following Møgelberg [17]) clearly need to be investigated. 


\section{Acknowledgements}

We gratefully acknowledge our discussions with Andreas Abel, Tadeusz Litak, Stefan Milius, Rasmus Møgelberg, Filip Sieczkowski, and Andrea Vezzosi, and the comments of the reviewers. This research was supported in part by the ModuRes Sapere Aude Advanced Grant from The Danish Council for Independent Research for the Natural Sciences (FNU). Aleš Bizjak is supported in part by a Microsoft Research PhD grant.

\section{References}

1. Abel, A., Pientka, B.: Wellfounded recursion with copatterns: A unified approach to termination and productivity. In: ICFP. pp. 185-196 (2013)

2. Abel, A., Vezzosi, A.: A formalized proof of strong normalization for guarded recursive types. In: APLAS. pp. 140-158 (2014)

3. Appel, A.W., Melliès, P.A., Richards, C.D., Vouillon, J.: A very modal model of a modern, major, general type system. In: POPL. pp. 109-122 (2007)

4. Atkey, R., McBride, C.: Productive coprogramming with guarded recursion. In: ICFP. pp. 197-208 (2013)

5. Bierman, G.M., de Paiva, V.C.: On an intuitionistic modal logic. Studia Logica 65(3), 383-416 (2000)

6. Birkedal, L., Møgelberg, R.E., Schwinghammer, J., Støvring, K.: First steps in synthetic guarded domain theory: step-indexing in the topos of trees. LMCS 8(4) (2012)

7. Birkedal, L., Schwinghammer, J., Støvring, K.: A metric model of lambda calculus with guarded recursion. In: FICS. pp. 19-25 (2010)

8. Bizjak, A., Birkedal, L., Miculan, M.: A model of countable nondeterminism in guarded type theory. In: RTA-TLCA. pp. 108-123 (2014)

9. Clouston, R., Goré, R.: Sequent calculus in the topos of trees. In: FoSSaCS (2015)

10. Coquand, T.: Infinite objects in type theory. In: TYPES. pp. 62-78 (1993)

11. Endrullis, J., Grabmayer, C., Hendriks, D.: Mix-automatic sequences (2013), Fields Workshop on Combinatorics on Words, contributed talk.

12. Giménez, E.: Codifying guarded definitions with recursive schemes. In: TYPES. pp. 39-59 (1995)

13. Hughes, J., Pareto, L., Sabry, A.: Proving the correctness of reactive systems using sized types. In: POPL. pp. 410-423 (1996)

14. Krishnaswami, N.R., Benton, N.: Ultrametric semantics of reactive programs. In: LICS. pp. 257-266 (2011)

15. McBride, C., Paterson, R.: Applicative programming with effects. J. Funct. Programming 18(1), 1-13 (2008)

16. Milius, S., Moss, L.S., Schwencke, D.: Abstract GSOS rules and a modular treatment of recursive definitions. LMCS 9(3) (2013)

17. Møgelberg, R.E.: A type theory for productive coprogramming via guarded recursion. In: CSL-LICS (2014)

18. Nakano, H.: A modality for recursion. In: LICS. pp. 255-266 (2000)

19. Prawitz, D.: Natural Deduction: A Proof-Theoretical Study. Dover Publ. (1965)

20. Rutten, J.J.M.M.: Behavioural differential equations: A coinductive calculus of streams, automata, and power series. Theor. Comput. Sci. 308(1-3), 1-53 (2003)

21. Severi, P.G., de Vries, F.J.J.: Pure type systems with corecursion on streams: from finite to infinitary normalisation. In: ICFP. pp. 141-152 (2012) 


\section{A Proofs for Section 3}

Proof (of Lem. 3.6). By induction on type formation, with $\rightarrow A$ case omitted,

a a base case, and $\mu \alpha$. $A$ considered only where $\alpha$ is not free in $A$.

Proof (of Lem. 3.8). By induction on the typing of $t$. We present the cases particular to our calculus.

next $t$ : case $i=1$ is trivial. $\llbracket \operatorname{next} t[\vec{t} / \vec{x}] \rrbracket_{i+1}(\gamma)=r_{i}^{\llbracket A \rrbracket} \circ \llbracket t[\vec{t} / \vec{x}] \rrbracket_{i+1}(\gamma)=r_{i}^{\llbracket A \rrbracket} \circ$ $\llbracket t \rrbracket_{i+1}\left(\llbracket t_{1} \rrbracket_{i+1}(\gamma), \ldots\right)$ by induction, which is $\llbracket$ next $t \rrbracket_{i+1}\left(\llbracket t_{1} \rrbracket_{i+1}(\gamma), \ldots\right)$.

$\llbracket(\operatorname{prev}[\vec{y} \leftarrow \vec{u}] . t)[\vec{t} / \vec{x}] \rrbracket_{i}(\gamma)=\llbracket \operatorname{prev}[\vec{y} \leftarrow \vec{u}[\vec{t} / \vec{x}]] . t \rrbracket_{i}(\gamma)$, which by definition is $\llbracket t \rrbracket_{i+1}\left(\llbracket u_{1}\left[\vec{t} / \vec{x} \rrbracket \rrbracket_{i}(\gamma), \ldots\right)=\llbracket t \rrbracket_{i+1}\left(\llbracket u_{1} \rrbracket_{i}\left(\llbracket t_{1} \rrbracket_{i}(\gamma), \ldots\right), \ldots\right)\right.$ by induction, which is $\llbracket \operatorname{prev}[\vec{y} \leftarrow \vec{u}] . t \rrbracket_{i}\left(\llbracket t_{1} \rrbracket_{i}(\gamma), \ldots\right)$.

$\llbracket \operatorname{box}[\vec{y} \leftarrow \vec{u}[\vec{t} / \vec{x}]] . t \rrbracket_{i}(\gamma)_{j}=\llbracket t \rrbracket_{j}\left(\llbracket u_{1}[\vec{t} / \vec{x}] \rrbracket_{i}(\gamma), \ldots\right)$, which by induction equals $\llbracket t \rrbracket_{j}\left(\llbracket u_{1} \rrbracket_{i}\left(\llbracket t_{1} \rrbracket_{i}(\gamma), \ldots\right), \ldots\right)=\llbracket \operatorname{box}\left[\vec{y} \leftarrow \vec{u} \rrbracket . t \rrbracket_{i}\left(\llbracket t_{1} \rrbracket_{i}(\gamma), \ldots\right)_{j}\right.$. $\llbracket$ unbox $t[\vec{t} / \vec{x}] \rrbracket_{i}(\gamma)=\llbracket t[\vec{t} / \vec{x}] \rrbracket_{i}(\gamma)_{i}=\llbracket t \rrbracket_{i}\left(\llbracket t_{1} \rrbracket_{i}(\gamma), \ldots\right)_{i}$ by induction, which is $\llbracket$ unbox $t \rrbracket_{i}\left(\llbracket t_{1} \rrbracket_{i}(\gamma), \ldots\right)$.

$u_{1} \circledast u_{2}$ : case $i=1$ is trivial. $\llbracket\left(u_{1} \circledast u_{2}\right)[\vec{t} / \vec{x}] \rrbracket_{i+1}(\gamma)=\left(\llbracket u_{1}[\vec{t} / \vec{x}] \rrbracket_{i+1}(\gamma)_{i}\right) \circ$ $\llbracket u_{2}\left[\vec{t} / \vec{x} \rrbracket \rrbracket_{i+1}(\gamma)=\left(\llbracket u_{1} \rrbracket_{i+1}\left(\llbracket t_{1} \rrbracket_{i+1}(\gamma), \ldots\right)_{i}\right) \circ \llbracket u_{2} \rrbracket_{i+1}\left(\llbracket t_{1} \rrbracket_{i+1}(\gamma), \ldots\right)\right.$, which is $\llbracket u_{1} \circledast u_{2} \rrbracket_{i+1}\left(\llbracket t_{1} \rrbracket_{i+1}(\gamma), \ldots\right)$.

Proof (of Soundness Thm. 3.9). We verify the reduction rules of Def. 2.2, extending this to any evaluation context, and to $\rightsquigarrow$, is easy. The product reduction case is standard, and function case requires Lem. 3.8, unfold fold is the application of mutually inverse arrows.

$\llbracket \operatorname{prev}[\vec{x} \leftarrow \vec{t}] . t \rrbracket_{i}=\llbracket t \rrbracket_{i+1}\left(\llbracket t_{1} \rrbracket_{i}, \ldots\right)$. Each $t_{k}$ is closed, so is denoted by an arrow from 1 to the constant $\mathcal{S}$-object $\llbracket A_{k} \rrbracket$, so by naturality $\llbracket t_{k} \rrbracket_{i}=\llbracket t_{k} \rrbracket_{i+1}$. But $\llbracket t \rrbracket_{i+1}\left(\llbracket t_{1} \rrbracket_{i+1}, \ldots\right)=\llbracket t\left[\vec{t} / \vec{x} \rrbracket_{i+1}\right.$ by Lem. 3.8, which is $\llbracket \operatorname{prev} t[\vec{t} / \vec{x}] \rrbracket_{i}$. $\llbracket$ prev next $t \rrbracket_{i}=\llbracket$ next $t \rrbracket_{i+1}=\llbracket t \rrbracket_{i}$.

$\llbracket \operatorname{unbox}(\operatorname{box}[\vec{x} \leftarrow \vec{t}] . t) \rrbracket_{i}=\left(\llbracket \operatorname{box}[\vec{x} \leftarrow \vec{t}] . t \rrbracket_{i}\right)_{i}=\llbracket t \rrbracket_{i}\left(\llbracket t_{1} \rrbracket_{i}, \ldots\right)=\llbracket t[\vec{t} / \vec{x}] \rrbracket_{i}$.

With $\circledast$-reduction, index 1 is trivial. $\llbracket$ next $t_{1} \circledast$ next $t_{2} \rrbracket_{i+1}=\left(\llbracket \text { next } t_{1} \rrbracket_{i+1}\right)_{i}$ 。 $\llbracket$ next $t_{2} \rrbracket_{i+1}=\left(r_{i}^{\llbracket A \rightarrow B \rrbracket} \circ \llbracket t_{1} \rrbracket_{i+1}\right)_{i} \circ r_{i}^{\llbracket A \rrbracket} \circ \llbracket t_{2} \rrbracket_{i+1}=\left(\llbracket t_{1} \rrbracket_{i} \circ r_{i}^{1}\right)_{i} \circ \llbracket t_{2} \rrbracket_{i} \circ r_{i}^{1}$ by naturality, which is $\left(\llbracket t_{1} \rrbracket_{i}\right)_{i} \circ \llbracket t_{2} \rrbracket_{i}=\llbracket t_{1} t_{2} \rrbracket_{i}=\llbracket t_{1} t_{2} \rrbracket_{i} \circ r_{i}^{1}=r_{i}^{\llbracket B \rrbracket} \circ \llbracket t_{1} t_{2} \rrbracket_{i+1}=$ $\llbracket \operatorname{next}\left(t_{1} t_{2}\right) \rrbracket_{i+1}$.

Proof (of Lem. 3.11). By induction on the construction of the type $A$.

$(i)$ follows with only interesting case the variable case $-A$ cannot be $\alpha$ because of the requirement that $\alpha$ be guarded in $A$.

(ii) follows with interesting cases: variable case enforces $b d(B)=0$; binary type-formers $\times, \rightarrow$ have for example bd $\left(A_{d}\right) \geq \operatorname{bd}\left(A_{1} \times A_{2}\right)$, so $\operatorname{bd}\left(A_{d}\right) \geq b d(B)$ and the induction follows; $\boldsymbol{\square}$ by construction has no free variables.

Lemma A.1. If $t \rightsquigarrow u$ and $a R_{i}^{A} u$ then $a R_{i}^{A} t$.

Proof. All cases follow similarly; consider $A_{1} \times A_{2} .\left(a_{1}, a_{2}\right) R_{i}^{A_{1} \times A_{2}} u$ implies $u \rightsquigarrow$ $\left\langle t_{1}, t_{2}\right\rangle$, where this value obeys some property. But then $t \rightsquigarrow\left\langle t_{1}, t_{2}\right\rangle$ similarly.

Lemma A.2. $a R_{i+1}^{A} t$ implies $r_{i}^{\llbracket A \rrbracket}(a) R_{i}^{A} t$. 
Proof. Cases $\mathbf{1}, \mathbf{N}$ are trivial. Case $\times$ follows by induction because restrictions are defined pointwise. Case $\mu$ follows by induction and the naturality of the isomorphism $h$. Case $A$ follows because $r_{i}^{\llbracket \square}(a)=a$.

For $A \rightarrow B$ take $j \leq i$ and $a^{\prime} R_{j}^{A} u$. By the downwards closure in the definition of $R_{i+1}^{A \rightarrow B}$ we have $f_{j}\left(a^{\prime}\right) R_{j}^{B} s[u / x]$. But $f_{j}=\left(r_{i}^{\llbracket A \rightarrow B \rrbracket}(f)\right)_{j}$.

With $\triangleright A$, case $i=1$ is trivial, so take $i=j+1 . a R_{j+2}^{A} t$ means $t \rightsquigarrow$ next $u$ and $a R_{j+1}^{A} u$, so by induction $r_{j}^{\llbracket A \rrbracket}(a) R_{j}^{A} u$, so $r_{j+1}^{\llbracket}(a) R_{j}^{A} u$ as required.

Lemma A.3. If $a R_{i}^{A} t$ and $A$ is constant, then $a R_{j}^{A} t$ for all $j$.

Proof. Easy induction on types, ignoring $\rightarrow A$ and treating $A$ as a base case.

We finally turn to the proof of the Fundamental Lemma.

Proof (of Lem. 3.13). By induction on the typing $\Gamma \vdash t: A$. \langle\rangle , zero cases are trivial, and $\left\langle u_{1}, u_{2}\right\rangle$, fold $t$ cases follow by easy induction.

succ: If $t[\vec{t} / \vec{x}]$ reduces to succ $^{l}$ zero for some $l$ then $\operatorname{succ} t[\vec{t} / \vec{x}]$ reduces to succ $^{l+1}$ zero, as we may reduce under the succ.

$\pi_{d} t$ : If $\llbracket t \rrbracket_{i}(\vec{a}) R_{i}^{A_{1} \times A_{2}} t[\vec{t} / \vec{x}]$ then $t[\vec{t} / \vec{x}] \rightsquigarrow\left\langle u_{1}, u_{2}\right\rangle$ and $u_{d}$ is related to the $d^{\prime}$ th projection of $\llbracket t \rrbracket_{i}(\vec{a})$. But then $\pi_{d} t[\vec{t} / \vec{x}] \rightsquigarrow \pi_{d}\left\langle u_{1}, u_{2}\right\rangle \mapsto u_{d}$, so Lem. A.1 completes the case.

$\lambda x . t$ : Taking $j \leq i$ and $a R_{j}^{A} u$, we must show that $\llbracket \lambda x . t \rrbracket_{i}(\vec{a})_{j}(a) R_{j}^{B} t[\vec{t} / \vec{x}][u / x]$. The left hand side is $\llbracket t \rrbracket_{j}\left(\vec{a} \Upsilon_{j}, a\right)$. For each $k, a_{k} \Upsilon_{j} R_{j}^{A_{k}} t_{k}$ by Lem. A.2, and induction completes the case.

$u_{1} u_{2}$ : By induction $u_{1}[\vec{t} / \vec{x}] \rightsquigarrow \lambda x . s$ and $\llbracket u_{1} \rrbracket_{k}(\vec{a})_{k}\left(\llbracket u_{2} \rrbracket_{k}(\vec{a})\right) R_{i}^{B} s\left[u_{2}[\vec{t} / \vec{x}] / x\right]$. Now $\left(u_{1} u_{2}\right) \rightsquigarrow(\lambda x . s)\left(u_{2}[\vec{t} / \vec{x}]\right) \mapsto s\left[u_{2}[\vec{t} / \vec{x}] / x\right]$, and Lem. A.1] completes.

unfold $t$ : we reduce under unfold, then reduce unfold fold, then use Lem. A.1. next $t$ : Trivial for index 1 . For $i=j+1$, if each $a_{k} R_{j+1}^{A_{k}} t_{k}$ then by Lem. A.2 $r_{j}^{\llbracket A_{k} \rrbracket}\left(a_{k}\right) R_{j}^{A_{k}} t_{k}$. Then by induction $\llbracket t \rrbracket_{j} \circ r_{j}^{\llbracket A_{1} \rrbracket \times \cdots \llbracket A_{m} \rrbracket}(\vec{a}) R_{j}^{A} t[\vec{t} / \vec{x}]$, whose left side is by naturality $r_{j}^{\llbracket A \rrbracket} \circ \llbracket t \rrbracket_{j+1}(\vec{a})=\llbracket \operatorname{next} t \rrbracket_{j+1}(\vec{a})$.

$\operatorname{prev}[\vec{y} \leftarrow \vec{u}] . t: \llbracket u_{k} \rrbracket_{i}(\vec{a}) R_{i}^{A_{k}} u_{k}[\vec{t} / \vec{x}]$ by induction, so $\llbracket u_{k} \rrbracket_{i}(\vec{a}) R_{i+1}^{A_{k}} u_{k}[\vec{t} / \vec{x}]$ by Lem. A.3. Then $\llbracket t \rrbracket_{i+1}\left(\llbracket u_{1} \rrbracket_{i}(\vec{a}), \ldots\right) R_{i+1}^{A} t\left[u_{1}[\vec{t} / \vec{x}] / y_{1}, \ldots\right]$ by induction, so we have $t\left[u_{1}[\vec{t} / \vec{x}] / y_{1}, \ldots\right] \rightsquigarrow$ next $s$ with $\llbracket t \rrbracket_{i+1}\left(\llbracket u_{1} \rrbracket_{k}(\vec{a}), \ldots\right) R_{i}^{A} s$. The left hand side is $\llbracket \operatorname{prev}[\vec{y} \leftarrow \vec{u}] . t \rrbracket_{i}(\vec{a})$, while $\operatorname{prev}[\vec{y} \leftarrow \vec{u}[\vec{t} / \vec{x}]] . t \mapsto \operatorname{prev} t\left[u_{1}[\vec{t} / \vec{x}] / y_{1}, \ldots\right] \rightsquigarrow$ prev next $s \mapsto s$, so Lem. A.1 completes.

box $[\vec{y} \leftarrow \vec{u}] . t$ : To show $\llbracket$ box $[\vec{y} \leftarrow \vec{u}] . t \rrbracket_{i}(\vec{a}) R_{i}^{\Pi^{A}}$ box $\left.[\vec{y} \leftarrow \vec{u}] . t\right)[\vec{t} / \vec{x}]$, we observe that the right hand side reduces in one step to box $t\left[u_{1}[\vec{t} / \vec{x}] / y_{1}, \ldots\right]$. The $j$ 'th element of the left hand side is $\llbracket t \rrbracket_{j}\left(\llbracket u_{1} \rrbracket_{k}(\vec{a}), \ldots\right)$. We need to show this is related by $R_{j}^{A}$ to $t\left[u_{1}[\vec{t} / \vec{x}] / y_{1}, \ldots\right]$; this follows by Lem. A.3 and induction.

unbox $t$ : By induction $t[\vec{t} / \vec{x}] \rightsquigarrow$ box $u$, so unbox $t[\vec{t} / \vec{x}] \rightsquigarrow$ unbox box $u \mapsto u$. By induction $\llbracket t \rrbracket_{i}(\vec{a})_{i} R_{i}^{A} u$, so $\llbracket$ unbox $t \rrbracket_{i}(\vec{a}) R_{i}^{A} u$, and Lem. A.1 completes.

$u_{1} \circledast u_{2}$ : Index 1 is trivial so set $i=j+1 . \llbracket u_{2} \rrbracket_{j+1}(\vec{a}) R_{j+1}^{A} u_{2}[\vec{t} / \vec{x}]$ implies $u_{2}[\vec{t} / \vec{x}] \rightsquigarrow$ next $s_{2}$ with $\llbracket u_{2} \rrbracket_{j+1}(\vec{a}) R_{j}^{A} s_{2}$. Similarly $u_{1} \rightsquigarrow$ next $s_{1}$ and $s_{1} \rightsquigarrow \lambda x . s$ with $\left(\llbracket u_{1} \rrbracket_{j+1}(\vec{a})_{j}\right) \circ \llbracket u_{2} \rrbracket_{j+1}(\vec{a}) R_{j}^{B} s\left[s_{2} / x\right]$. The left hand side is exactly $\llbracket u_{1} \circledast$ 
$u_{2} \rrbracket_{j+1}(\vec{a})$. Now $u_{1} \circledast u_{2} \rightsquigarrow$ next $s_{1} \circledast u_{2} \rightsquigarrow$ next $s_{1} \circledast$ next $s_{2} \mapsto \operatorname{next}\left(s_{1} s_{2}\right)$, and $s_{1} s_{2} \rightsquigarrow(\lambda x . s) s_{2} \mapsto s\left[s_{2} / x\right]$, completing the proof.

\section{B Example Proofs in $\operatorname{Lg} \lambda$}

We first record a substitution property of box and prev for later use.

Lemma B.1. Let $A_{1}, \ldots, A_{k}$ and $B$ be constant types and $C$ any type. If we have $x: B \vdash t: C$ and $y_{1}: A_{k}, \ldots, y_{k}: A_{k} \vdash t^{\prime}: B$ then

$$
\text { box }\left[x \leftarrow t^{\prime}\right] . t=\mathbf{m}_{C} \text { box } \iota . t\left[t^{\prime} / x\right] .
$$

If $C=\triangleright D$ then we also have

$$
\operatorname{prev}\left[x \leftarrow t^{\prime}\right] . t={ }_{D} \text { prev } \iota . t\left[t^{\prime} / x\right]
$$

We can prove the first part of the lemma in the logic, using Prop. 4.2 and the $\beta$-rule for box. We can also prove the second part of the lemma for total and inhabited types $D$ with the rules we have stated so far using the $\beta$-rule for next. For arbitrary $D$ we can prove the lemma using the semantics.

\section{B.1 Acausal Example}

To see that Löb induction can be used to prove properties of recursively defined acausal functions we show that for any $n: \mathbf{N}$ and any $f: \mathbf{N} \rightarrow \mathbf{N}$ we have

$$
\text { every2nd (box } \iota \text {. iterate }(\text { next } f) n)=\operatorname{strg} \text { iterate }\left(\text { next } f^{2}\right) n,
$$

where we write $f^{2}$ for $\lambda n . f(f n)$. We first derive the intermediate result

$$
\forall m: \mathbf{N}, \mathrm{tl}(\text { box } \iota \text {. iterate }(\text { next } f) m)=\text { str box } \iota \text {. iterate }(\text { next } f)(f m),
$$

by unfolding and applying Prop. 4.3 .

$$
\begin{aligned}
\mathrm{tl}(\operatorname{box} \iota . \text { iterate }(\operatorname{next} f) m) & =\operatorname{box}[s \leftarrow \operatorname{box} \iota \cdot \text { iterate }(\operatorname{next} f) m] \cdot \operatorname{prev} \iota \cdot \mathrm{t}^{\mathrm{g}}(\operatorname{unbox} s) \\
& =\operatorname{box} \iota \cdot \operatorname{prev} \iota \cdot \mathrm{t}^{\mathrm{g}}(\text { iterate }(\operatorname{next} f) m) \quad(\operatorname{by~Lem} . \text { B.1) } \\
& =\operatorname{box} \iota \cdot \operatorname{prev} \iota \cdot \operatorname{next}(\operatorname{iterate}(\operatorname{next} f)(f m)) \\
& =\operatorname{box} \iota \cdot \text { iterate }(\operatorname{next} f)(f m) .
\end{aligned}
$$

Now assume

$$
\left.\triangleright(\forall n: \mathbf{N}, \text { every2nd(box } \iota \text {. iterate }(\text { next } f) n)={ }_{\operatorname{Str}} \text { iterate }\left(\text { next } f^{2}\right) n\right),
$$

then by Löb induction we can derive

$$
\begin{aligned}
\text { every2nd }(\text { box } \iota \text {. iterate }(\operatorname{next} f) n) \\
=n:: \operatorname{next}(\operatorname{every} 2 \mathrm{nd}(\mathrm{tl}(\mathrm{tl}(\text { box } \iota \text {. iterate }(\operatorname{next} f) n)))) \\
=n:: \operatorname{next}(\operatorname{every} 2 \mathrm{nd}(\operatorname{box} \iota \text {. iterate }(\text { next } f)(f(f n)))) \quad(\text { by } 2) \\
=n:: \operatorname{next}\left(\text { iterate }\left(\operatorname{next} f^{2}\right)(f(f n))\right) \quad\left(\text { by } 3 \text { and } \mathrm{EQ}_{\text {next }}^{\triangleright}\right) \\
=i \text { iterate }\left(\operatorname{next} f^{2}\right) n .
\end{aligned}
$$




\section{B.2 Higher-Order Logic Example}

We now prove

$$
\begin{aligned}
& \forall P, Q:(\mathbf{N} \rightarrow \Omega), \forall f: \mathbf{N} \rightarrow \mathbf{N},(\forall x: \mathbf{N}, P(x) \Rightarrow Q(f(x))) \\
& \Rightarrow \forall x s: \operatorname{Str}, P_{\operatorname{Str}}(x s) \Rightarrow Q_{\operatorname{Str}}\left(\operatorname{map}^{\mathrm{g}} f x s\right) .
\end{aligned}
$$

This is a simple property of map ${ }^{\mathrm{g}}$, but the proof shows how the pieces fit together. Recall that map satisfies map $f x s=f\left(\operatorname{hd}^{\mathrm{g}} x s\right)::\left(\operatorname{next}\left(\operatorname{map}^{\mathrm{g}} f\right) \circledast\left(\mathrm{t}^{\mathrm{g}} x s\right)\right)$. We prove the property by Löb induction. So let $P$ and $Q$ be predicates on $\mathbf{N}$ and $f$ a function on $\mathbf{N}$ that satisfies $\forall x: \mathbf{N}, P(x) \Rightarrow Q(f(x))$. To use Löb induction assume

$$
\triangleright\left(\forall x s: \operatorname{Str}, P_{\operatorname{Str}}(x s) \Rightarrow Q_{\operatorname{Str}^{g}}\left(\operatorname{map}^{\mathrm{g}} f x s\right)\right)
$$

and let $x s$ be a stream satisfying $P_{\mathrm{Str}}$. Unfolding $P_{\mathrm{Str}}(x s)$ we get $P\left(\mathrm{hd}^{\mathrm{g}} x s\right)$ and lift $\left(\right.$ next $\left.P_{\text {Strg }} \circledast\left(\mathrm{tl}^{\mathrm{g}} x s\right)\right)$ and we need to prove $Q\left(\mathrm{hd}^{\mathrm{g}}\left(\operatorname{map}^{\mathrm{g}} f x s\right)\right)$ and also lift $\left(\right.$ next $\left.Q_{\text {Strg }} \circledast\left(\mathrm{t}^{\mathrm{g}}\left(\operatorname{map}^{\mathrm{g}} f x s\right)\right)\right)$. The first is easy since $Q\left(\mathrm{hd}^{\mathrm{g}}\left(\operatorname{map}^{\mathrm{g}} f x s\right)\right)=$ $Q\left(f\left(\mathrm{hd}^{\mathrm{g}} x s\right)\right)$. For the second we have $\mathrm{tl}^{\mathrm{g}}\left(\operatorname{map}^{\mathrm{g}} f x s\right)=\operatorname{next}(\operatorname{map} \mathrm{g} f) \circledast\left(\mathrm{t}^{\mathrm{g}} x s\right)$. Since Str is a total and inhabited type there is a stream $x s^{\prime}$ such that next $x s^{\prime}=$ $\mathrm{t}^{\mathrm{g}} x s$. This gives $\mathrm{t}^{\mathrm{g}}\left(\operatorname{map}^{\mathrm{g}} f x s\right)=\operatorname{next}\left(\operatorname{map}^{\mathrm{g}} f x s^{\prime}\right)$ and so our desired result reduces to lift $\left(\operatorname{next}\left(Q_{\mathrm{Strg}}\left(\operatorname{map}^{\mathrm{g}} f x s^{\prime}\right)\right)\right)$ and lift $\left(\right.$ next $\left.P_{\mathrm{Str}} \circledast\left(\mathrm{t}^{\mathrm{g}} x s\right)\right)$ is equivalent to $\operatorname{lift}\left(\operatorname{next}\left(P_{\mathrm{Str}}\left(x s^{\prime}\right)\right)\right)$. Now lift $\circ$ next $=\triangleright$ and so what we have to prove is $\triangleright\left(Q_{\operatorname{Str}}\left(\operatorname{map}^{\mathrm{g}} f x s^{\prime}\right)\right)$ from $\triangleright\left(P_{\operatorname{Str}^{g}}\left(x s^{\prime}\right)\right)$, which follows directly from the induction hypothesis (4).

\section{Sums}

This appendix extends Secs. 2, 3 and 4 to add sum types to the $\mathrm{g} \lambda$-calculus. and to $\operatorname{logic} L g \lambda$.

Binary sums in Atkey and McBride 4 come with the type isomorphism

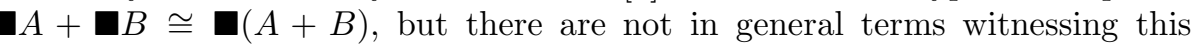
isomorphism. Likewise if binary sums are added to our calculus as obvious we may define the term

$$
\lambda x \text {. box } \iota \text {. case } x \text { of } x_{1} \text {. in } \text { in }_{1} \text { unox } x_{1} ; x_{2} . \text { in }_{2} \text { unbox } x_{2}: \mathbf{\square} A+\mathbf{\square} B \rightarrow(A+B)
$$

but no inverse is definable in general. We believe such a map may be useful when working with guarded recursive types involving sum, such as the type of potentially infinite lists, and in any case the isomorphism is valid in the topos of trees and so it is harmless for us to reflect this in our calculus. We do this via a new term-former box ${ }^{+}$allowing us to define

$$
\lambda x \text {. box }{ }^{+} \iota \text {. unbox } x: \mathbf{\square}(A+B) \rightarrow \mathbf{\square} A+\mathbf{\square} B
$$

This construct may be omitted without effecting the results of this section. 
Definition C.1 (ref. Defs. 2.1||2.2|2.3|2.4|2.7|2.8). g $\lambda$-terms are given by the grammar

$$
t::=\cdots \mid \text { abort } t\left|\operatorname{in}_{d} t\right| \text { case } t \text { of } x_{1} . t ; x_{2} . t \mid \text { box }{ }^{+} \sigma . t
$$

where $d \in\{1,2\}$, and $x_{1}, x_{2}$ are variables. We abbreviate terms with box ${ }^{+}$as for prev and box.

The reduction rules on closed $\mathrm{g} \lambda$-terms with sums are

$$
\begin{aligned}
\text { case in }_{d} t \text { of } x_{1} \cdot t_{1} ; x_{2} \cdot t_{2} & \mapsto t_{d}\left[t / x_{d}\right] & & (d \in\{1,2\}) \\
\text { box }^{+}[\vec{x} \leftarrow \vec{t}] . t & \left.\mapsto \text { box }^{+} t \vec{t} / \vec{x}\right] & & (\vec{x} \text { non-empty }) \\
\text { box }^{+} \operatorname{in}_{i} t & \mapsto \operatorname{in}_{i} \text { box } t & &
\end{aligned}
$$

Values are terms of the form

$$
\cdots\left|\mathrm{in}_{1} t\right| \mathrm{in}_{2} t
$$

Evaluation contexts are defined by the grammar

$$
E::=\cdots \mid \text { abort } E \mid \text { case } E \text { of } x_{1} \cdot t_{1} ; x_{2} \cdot t_{2} \mid \text { box }^{+} E
$$

g $\lambda$-types for sums are defined inductively by the rules of Fig. 5, and the new typing judgments are given in Fig. [6, where $d \in\{1,2\}$.

$$
\overline{\nabla \vdash \mathbf{0}} \quad \frac{\nabla \vdash A_{1} \quad \nabla \vdash A_{2}}{\nabla \vdash A_{1}+A_{2}}
$$

Fig. 5. Type formation for sums in the $g \lambda$-calculus

$$
\begin{aligned}
& \frac{\Gamma \vdash t: \mathbf{0}}{\Gamma \vdash \text { abort } t: A} \quad \frac{\Gamma \vdash t: A_{d}}{\Gamma \vdash \operatorname{in}_{d} t: A_{1}+A_{2}} \\
& \frac{\Gamma \vdash t: A_{1}+A_{2} \quad \Gamma, x_{1}: A_{1} \vdash t_{1}: A \quad \Gamma, x_{2}: A_{2} \vdash t_{2}: A}{\Gamma \vdash \operatorname{case} t \text { of } x_{1} \cdot t_{1} ; x_{2} . t_{2}: A} \\
& x_{1}: A_{1}, \ldots, x_{n}: A_{n} \vdash t: B_{1}+B_{2} \\
& \frac{\Gamma \vdash t_{1}: A_{1} \quad \cdots \quad \Gamma \vdash t_{n}: A_{n}}{\Gamma \vdash \text { box }^{+}\left[x_{1} \leftarrow t_{1}, \ldots, x_{n} \leftarrow t_{n}\right] . t: \mathbf{\square} B_{1}+\mathbf{\square} B_{2}} A_{1}, \ldots, A_{n} \text { constant }
\end{aligned}
$$

Fig. 6. Typing rules for sums in the $g \lambda$-calculus 
We now consider denotational semantics. Note that the initial object of $\mathcal{S}$ is $\Delta \emptyset$ (ref. Def. 3.2), while binary coproducts in $\mathcal{S}$ are defined pointwise. By naturality it holds that for any arrow $f: X \rightarrow Y+Z$ and $x \in X, f_{i}(x)$ must be an element of the same side of the sum for all $i$.

Definition C.2 (ref. Defs. 3.3/3.7).

- $\llbracket \mathbf{0} \rrbracket$ is the constant functor $\Delta \emptyset$;

- $\llbracket A_{1}+A_{2} \rrbracket(\vec{W})=\llbracket A_{1} \rrbracket(\vec{W})+\llbracket A_{2} \rrbracket(\vec{W})$ and likewise for $\mathcal{S}$-arrows.

Term-formers for sums are intepreted via $\mathcal{S}$-coproducts, with abort, $\mathrm{in}_{d}$ and case defined as usual, and box ${ }^{+}$defined as follows.

- Let $\llbracket t \rrbracket_{j}\left(\llbracket t_{1} \rrbracket_{i}(\gamma), \ldots, \llbracket t_{n} \rrbracket_{i}(\gamma)\right)$ (which is well-defined by Lem. 3.6) be $\left[a_{j}, d\right]$ as $j$ ranges, recalling that $d \in\{1,2\}$ is the same for all $i$. Define $a: 1 \rightarrow \llbracket A_{d} \rrbracket$ to have $j$ 'th element $a_{j}$. Then $\llbracket$ box $^{+}[\vec{x} \leftarrow \vec{t}] . t \rrbracket_{i}(\gamma) \triangleq[a, d]$.

We now proceed to the sum cases of our proofs.

Proof (box ${ }^{+}[\vec{y} \leftarrow \vec{u}] . t$ case of Lem. [3.8)). By induction we have $\llbracket u_{k}[\vec{t} / \vec{x}] \rrbracket_{i}(\gamma)=$ $\llbracket u_{k} \rrbracket_{i}\left(\llbracket t_{1} \rrbracket_{i}(\gamma), \ldots\right)$. Hence $\llbracket t \rrbracket_{j}\left(\llbracket u_{1}\left[\vec{t} / \vec{x} \rrbracket \rrbracket_{i}(\gamma), \ldots\right)=\llbracket t \rrbracket_{j}\left(\llbracket u_{1} \rrbracket_{i}\left(\llbracket t_{1} \rrbracket_{i}(\gamma), \ldots\right), \ldots\right)\right.$ as required.

Proof (box ${ }^{+}$cases of Soundness Thm. 3.9). Because each $\llbracket A_{k} \rrbracket$ is a constant object (Lem. [3.6), $\llbracket t_{k} \rrbracket_{i}=\llbracket t_{k} \rrbracket_{j}$ for all $i, j$. Hence $\llbracket$ box $^{+}\left[\vec{x} \leftarrow \vec{t} \rrbracket_{t} \rrbracket_{i}\right.$ is defined via components $\llbracket t \rrbracket_{j}\left(\llbracket t_{1} \rrbracket_{j}, \ldots\right)$ and $\llbracket$ box $^{+} t[\vec{t} / \vec{x}] \rrbracket$ is defined via components $\llbracket t\left[\vec{t} / \vec{x} \rrbracket_{j}\right.$. These are equal by Lem 3.8 .

$\llbracket$ box ${ }^{+} \mathrm{in}_{d} t \rrbracket_{i}$ is the $d^{\prime}$ 'th injection into the function with $j$ 'th component $\llbracket t \rrbracket_{j}$, and likewise for $\llbracket \operatorname{in}_{d}$ box $t \rrbracket_{i}$.

Definition C.3 (ref. Def. 3.12).

- $[a, d] R_{i}^{A_{1}+A_{2}} t$ iff $t \rightsquigarrow \operatorname{in}_{d} u$ for $d=1$ or 2 , and $a R_{i}^{A_{d}} u$.

Note that $R_{i}^{\mathbf{0}}$ is (necessarily) everywhere empty.

Proof (for Lems. A.1 and A.2). For $\mathbf{0}$ cases the premise fails so the the lemmas are vacuous. + cases follow as for $\times$.

Proof (ref. Fundamental Lemma 3.13). abort: The induction hypothesis states that $\llbracket t \rrbracket_{k}(\vec{a}) R_{k}^{0} t[\vec{t} / \vec{x}]$, but this is not possible, so the theorem holds vacuously.

$\operatorname{in}_{d} t$ case follows by easy induction.

case $t$ of $y_{1} \cdot u_{1} ; y_{2} . u_{2}$ : If $\llbracket t \rrbracket_{i}(\vec{a}) R_{i}^{A_{1}+A_{2}} t\left[\vec{t} / \vec{x} \rrbracket\right.$ then $t[\vec{t} / \vec{x}] \rightsquigarrow \mathrm{in}_{d} u$ for some $d \in\{1,2\}$, with $\llbracket t \rrbracket_{i}(\vec{a})=[a, d]$ and $a R_{i}^{A_{d}} u$. Then $\llbracket u_{d} \rrbracket_{i}(\vec{a}, a) R_{k}^{A} u_{d}\left[\vec{t} / \vec{x}, u / y_{d}\right]$.

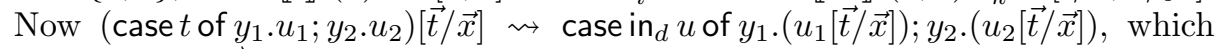
reduces to $u_{d}\left[\vec{t} / \vec{x}, u / y_{i}\right]$, and Lem. A.1 completes.

box $^{+}[\vec{y} \leftarrow \vec{u}] . t: \llbracket u_{k} \rrbracket_{i}(\vec{a}) R_{i}^{A_{k}} u_{k}[\vec{t} / \vec{x}]$ by induction, so $\llbracket u_{k} \rrbracket_{i}(\vec{a}) R_{j}^{A_{k}} u_{k}[\vec{t} / \vec{x}]$ for any $j$ by Lem. A.3, By induction $\llbracket t \rrbracket_{j}\left(\llbracket u_{1} \rrbracket_{k}(\vec{a}), \ldots\right) R_{j}^{B_{1}+B_{2}} t\left[u_{1}[\vec{t} / \vec{x}] / y_{1}, \ldots\right]$. If $\llbracket t \rrbracket_{j}\left(\llbracket u_{1} \rrbracket_{k}(\vec{a}), \ldots\right)$ is some $\left[b_{j}, d\right]$ we have $t\left[u_{1}[\vec{t} / \vec{x}] / y_{1}, \ldots\right] \rightsquigarrow \operatorname{in}_{d} s$ with $b_{j} R_{j}^{B_{d}} s$. Now $\left(\operatorname{box}^{+}[\vec{y} \leftarrow \vec{u}] . t\right)[\vec{t} / \vec{x}] \mapsto$ box $^{+} t\left[u_{1}[\vec{t} / \vec{x}] / y_{1}, \ldots\right] \rightsquigarrow$ box $^{+}$in $_{d} s$, which finally reduces to in box $_{s}$, which yields the result. 
The logic $L \mathrm{~g} \lambda$ may be extended to sums via the usual $\beta \eta$-laws and commuting conversions for binary sums and the equational version of the box ${ }^{+}$rule (ref. Fig. (3):

$$
\frac{\Gamma \cdot t: B_{d} \quad \Gamma \vdash \vec{t}: \Gamma_{\mathbf{\square}}}{\Gamma \vdash \operatorname{box}^{+}[\vec{x} \leftarrow \vec{t}] .\left(\operatorname{in}_{d} t\right)=\operatorname{in}_{d}(\operatorname{box}[\vec{x} \leftarrow \vec{t}] \cdot t)}
$$

\section{Proof of Definability of Solutions of Behavioural Differential Equations in $\mathbf{g} \boldsymbol{\lambda}$}

An equivalent presentation of the topos of trees is as sheaves over $\omega$ (with Alexandrov topology) Sh $(\omega)$. In this section it is more convenient to work with sheaves than with presheaves because the global sections functor $I^{1}$ in the sequence of adjoints

$$
\Pi_{1} \dashv \Delta \dashv \Gamma
$$

where

$$
\begin{array}{rrr}
\Pi_{1}: \mathcal{S} \rightarrow \text { Set } & : \text { Set } \rightarrow \mathcal{S} & \Gamma: \mathcal{S} \rightarrow \text { Set } \\
\Pi_{1}(X)=X(1) & \Delta(a)(\alpha)=\left\{\begin{array}{lll}
1 & \text { if } \alpha=0 & \Gamma(X)=X(\omega) \\
a & \text { otherwise }
\end{array}\right.
\end{array}
$$

is just evaluation at $\omega$, i.e. the limit is already present. This simplifies notation. Another advantage is that $\boldsymbol{\mathcal { S }} \rightarrow \mathcal{S}$ is given as

$$
\begin{aligned}
(\triangleright X)(\nu+1) & =X(\nu) \\
(\triangleright X)(\alpha) & =X(\alpha)
\end{aligned}
$$

where $\alpha$ is a limit ordinal (either 0 or $\omega$ ) which means that $\rightarrow(\omega)=X(\omega)$ and as a consequence, next $_{\omega}=\operatorname{id}_{X(\omega)}$ and $\Gamma(-X)=\Gamma(X)$ for any $X \in \mathcal{S}$ and so $\boldsymbol{\square}(\boldsymbol{\nabla})=\mathbf{\square} X$ for any $X$ so we don't have to deal with mediating isomorphisms.

First we have a simple statement, but useful later, since it gives us a precise goal to prove later when considering the interpretation.

Lemma D.1. Let $X, Y$ be objects of $\mathcal{S}$. Let $F:\left(Y^{X}\right) \rightarrow Y^{X}$ be a morphism in $\mathcal{S}$ and $\underline{F}$ a function in Set from $Y(\omega)^{X(\omega)}$ to $Y(\omega)^{X(\omega)}$. Suppose that the diagram

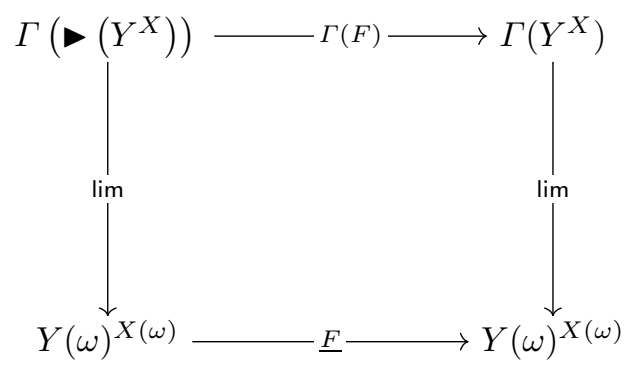

\footnotetext{
${ }^{1}$ This standard notation for this functor should not to be confused with our notation for typing contexts.
} 
where $\lim \left(\left\{g_{\nu}\right\}_{\nu=0}^{\omega}\right)=g_{\omega}$ commutes. By Banach's fixed point theorem $F$ has a unique fixed point, say $u: 1 \rightarrow Y^{X}$.

Then $\lim (\Gamma(u)(*))=\lim (\Gamma($ next $\circ u)(*))=\Gamma($ next $\circ u)(*)_{\omega}=u_{\omega}(*)_{\omega}$ is a fixed point of $\underline{F}$.

Proof. The proof is trivial.

$$
\begin{aligned}
\underline{F}(\lim (\Gamma(u)(*))) & =\lim (\Gamma(F)(\Gamma(\text { next } \circ u)(*))) \\
& =\lim (\Gamma(F \circ \text { next } \circ u)(*))=\lim (\Gamma(u)(*)) .
\end{aligned}
$$

Note that lim is not an isomorphism. There are (in general) many more functions from $X(\omega)$ to $Y(\omega)$ than those that arise from natural transformations. The ones that arise from natural transformations are the non-expansive ones.

\section{D.1 Behavioural Differential Equations}

Let $\Sigma_{A}$ be a signature of function symbols with two types, $A$ and Str. Suppose we wish to define a new $k$-ary operation given the signature $\Sigma_{A}$. We need to provide two terms $h_{f}$ and $t_{f}$ (standing for head and tail). $h_{f}$ has to be a term using function symbols in signature $\Sigma_{A}$ and have type

$$
x_{1}: A, x_{2}: A, \cdots, x_{k}: A \vdash h_{f}: A
$$

and $t_{f}$ has to be a term in the signature extended with a new function symbol $f$ of type $(\mathrm{Str})^{k} \rightarrow \mathrm{Str}$ and have type

$$
x_{1}: A, \cdots, x_{k}: A, y_{1}: \operatorname{Str}, \cdots, y_{k}: \operatorname{Str}, z_{1}: \operatorname{Str}, \cdots, z_{k}: \operatorname{Str} \vdash t_{f}: \operatorname{Str}
$$

In the second term the variables $x$ (intuitively) denote the head elements of the streams, the variables $y$ denote the streams, and the variables $z$ denote the tails of the streams.

We now define two interpretations of $h_{f}$ and $t_{f}$. First in the topos of trees and then in Set.

We choose a set $a \in$ Set and define $\llbracket A \rrbracket_{\mathcal{S}}=\Delta(a)$ and $\llbracket \operatorname{Str} \rrbracket_{\mathcal{S}}=\mu X . \Delta(a) \times$ - $(X)$. To each function symbol $g \in \Sigma$ of type $\tau_{1}, \ldots, \tau_{n} \rightarrow \tau_{n+1}$ we assign a morphism

$$
\llbracket g \rrbracket_{\mathcal{S}}: \llbracket \tau_{1} \rrbracket_{\mathcal{S}} \times \llbracket \tau_{2} \rrbracket_{\mathcal{S}} \times \cdots \times \llbracket \tau_{n} \rrbracket_{\mathcal{S}} \rightarrow \llbracket \tau_{n+1} \rrbracket_{\mathcal{S}} .
$$

Then we define the interpretation of $h_{f}$ by induction as a morphism of type $\llbracket A \rrbracket_{\mathcal{S}}^{k} \rightarrow \llbracket A \rrbracket_{\mathcal{S}}$ by

$$
\begin{aligned}
\llbracket x_{i} \rrbracket_{\mathcal{S}} & =\pi_{i} \\
\llbracket g\left(t_{1}, t_{2}, \ldots, t_{n}\right) \rrbracket_{\mathcal{S}} & =\llbracket g \rrbracket_{\mathcal{S}} \circ\left\langle\llbracket t_{1} \rrbracket_{\mathcal{S}}, \llbracket t_{2} \rrbracket_{\mathcal{S}}, \cdots, \llbracket t_{n} \rrbracket_{\mathcal{S}}\right\rangle .
\end{aligned}
$$

For $t_{f}$ we interpret the types and function symbols in $\Sigma_{A}$ in the same way. But recall that $t_{f}$ also contains a function symbol $f$. So the denotation of $t_{f}$ will be a morphism with the following type

$$
\llbracket t_{f} \rrbracket_{\mathcal{S}}: \triangleright\left(\llbracket \operatorname{Str} \rrbracket_{\mathcal{S}}^{\llbracket \operatorname{Str} \rrbracket_{\mathcal{S}}^{k}}\right) \times \llbracket A \rrbracket_{\mathcal{S}}^{k} \times \llbracket \operatorname{Str} \rrbracket_{\mathcal{S}}^{k} \times\left(\triangleright\left(\llbracket \operatorname{Str} \rrbracket_{\mathcal{S}}\right)\right)^{k} \rightarrow\left(\llbracket \operatorname{Str} \rrbracket_{\mathcal{S}}\right)
$$


and is defined as follows

$$
\begin{aligned}
\llbracket x_{i} \rrbracket_{\mathcal{S}} & =\text { next } \circ \iota \circ \pi_{x_{i}} \\
\llbracket y_{i} \rrbracket_{\mathcal{S}} & =\text { next } \circ \pi_{y_{i}} \\
\llbracket z_{i} \rrbracket_{\mathcal{S}} & =\pi_{z_{i}} \\
\llbracket g\left(t_{1}, t_{2}, \ldots, t_{n}\right) \rrbracket_{\mathcal{S}} & =\boldsymbol{}\left(\llbracket g \rrbracket_{\mathcal{S}}\right) \circ \mathbf{c a n} \circ\left\langle\llbracket t_{1} \rrbracket_{\mathcal{S}}, \llbracket t_{2} \rrbracket_{\mathcal{S}}, \cdots, \llbracket t_{n} \rrbracket_{\mathcal{S}}\right\rangle \quad \text { if } g \neq f \\
\llbracket f\left(t_{1}, t_{2}, \ldots, t_{k}\right) \rrbracket_{\mathcal{S}} & =\text { eval } \circ\left\langle J \circ \pi_{f}, \text { can } \circ\left\langle\llbracket t_{1} \rrbracket_{\mathcal{S}}, \llbracket t_{2} \rrbracket_{\mathcal{S}}, \cdots, \llbracket t_{k} \rrbracket_{\mathcal{S}}\right\rangle\right\rangle
\end{aligned}
$$

where can is the canonical isomorphism witnessing that preserves products, eval is the evaluation map and $\iota$ is the suitably encoded morphism that when given $a$ constructs the stream with head $a$ and tail all zeros. This exists and is easy to construct.

Next we define the denotation of $h_{f}$ and $t_{f}$ in Set. We set $\llbracket A \rrbracket_{\text {Set }}=a$ and $\llbracket \operatorname{Str} \rrbracket_{\text {Set }}=\llbracket \operatorname{Str} \rrbracket_{\mathcal{S}}(\omega)$. For each function symbol in $\Sigma_{A}$ we define $\llbracket g \rrbracket_{\text {Set }}=$ $\Gamma \llbracket g \rrbracket_{\mathcal{S}}=\left(\llbracket g \rrbracket_{\mathcal{S}}\right)_{\omega}$.

We then define $\llbracket h_{f} \rrbracket_{\text {Set }}$ as a function

$$
\llbracket A \rrbracket_{\text {Set }}^{k} \rightarrow \llbracket A \rrbracket_{\text {Set }}
$$

exactly the same as we defined $\llbracket h_{f} \rrbracket_{\mathcal{S}}$.

$$
\begin{aligned}
\llbracket x_{i} \rrbracket_{\text {Set }} & =\pi_{i} \\
\llbracket g\left(t_{1}, t_{2}, \ldots, t_{n}\right) \rrbracket_{\text {Set }} & =\llbracket g \rrbracket_{\text {Set }} \circ\left\langle\llbracket t_{1} \rrbracket_{\text {Set }}, \llbracket t_{2} \rrbracket_{\text {Set }}, \cdots, \llbracket t_{n} \rrbracket_{\text {Set }}\right\rangle .
\end{aligned}
$$

The denotation of $t_{f}$ is somewhat different in the way that we do not guard the tail and the function being defined with a $\boldsymbol{\bullet}$. We define

$$
\llbracket t_{f} \rrbracket_{\text {Set }}: \llbracket \operatorname{Str} \rrbracket_{\text {Set }}^{\llbracket \mathrm{Strt}} \rrbracket_{\text {Set }}^{k} \times \llbracket A \rrbracket_{\text {Set }}^{k} \times \llbracket \mathrm{Str} \rrbracket_{\text {Set }}^{k} \times\left(\llbracket \mathrm{Str} \rrbracket_{\text {Set }}\right)^{k} \rightarrow \llbracket \mathrm{Str} \rrbracket_{\text {Set }}
$$

as follows

$$
\begin{aligned}
\llbracket x_{i} \rrbracket_{\text {Set }} & =\iota \circ \pi_{x_{i}} \\
\llbracket y_{i} \rrbracket_{\text {Set }} & =\pi_{y_{i}} \\
\llbracket z_{i} \rrbracket_{\text {Set }} & =\pi_{z_{i}} \\
\llbracket g\left(t_{1}, t_{2}, \ldots, t_{n}\right) \rrbracket_{\text {Set }} & =\llbracket g \rrbracket_{\text {Set }} \circ\left\langle\llbracket t_{1} \rrbracket_{\text {Set }}, \llbracket t_{2} \rrbracket_{\text {Set }}, \cdots, \llbracket t_{n} \rrbracket_{\text {Set }}\right\rangle \quad \text { if } g \neq f \\
\llbracket f\left(t_{1}, t_{2}, \ldots, t_{k}\right) \rrbracket_{\text {Set }} & =\text { eval } \circ\left\langle\pi_{f},\left\langle\llbracket t_{1} \rrbracket_{\text {Set }}, \llbracket t_{2} \rrbracket_{\text {Set }}, \cdots, \llbracket t_{k} \rrbracket_{\text {Set }}\right\rangle\right\rangle
\end{aligned}
$$

where $\iota$ is again the same operation, this time on actual streams in Set.

We then define

$$
\underline{F}: \llbracket \operatorname{Str} \rrbracket_{\text {Set }}^{\llbracket \mathrm{Str} \rrbracket} \rrbracket_{\text {Set }}^{k} \rightarrow \llbracket \mathrm{Str} \rrbracket_{\text {Set }}^{\llbracket \mathrm{Str} \rrbracket_{\text {Set }}^{k}}
$$

as

$$
\underline{F}(\phi)(\vec{\sigma})=\Gamma(\text { fold })\left(\left(\llbracket h_{f} \rrbracket_{\text {Set }}(\mathbf{h d}(\vec{\sigma})), \llbracket t_{f} \rrbracket_{\text {Set }}(\phi, \mathbf{h d}(\vec{\sigma}), \vec{\sigma}, \mathbf{t l}(\vec{\sigma}))\right)\right)
$$


where hd and $\mathbf{t l}$ are head and tail functions (extended in the obvious way to tuples). Here fold is the isomorphism witnessing that guarded streams are indeed the fixed point of the defining functor.

Similarly we define

$$
F: \triangleright\left(\llbracket \operatorname{Str} \rrbracket_{\mathcal{S}}^{\llbracket \operatorname{Str} \rrbracket_{\mathcal{S}}^{k}}\right) \rightarrow \llbracket \operatorname{Str} \rrbracket_{\mathcal{S}}^{\llbracket \operatorname{Str} \rrbracket_{\mathcal{S}}^{k}}
$$

as the exponential transpose $\Lambda$ of

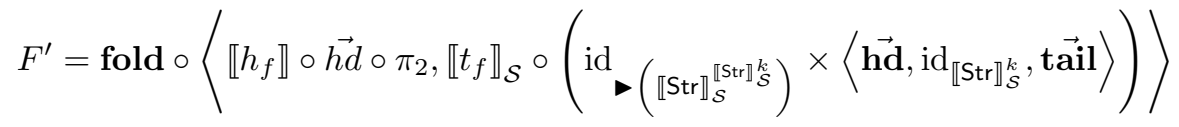

Proposition D.2. For the above defined $F$ and $\underline{F}$ we have

$$
\lim \circ \Gamma(F)=\underline{F} \circ \lim
$$

Proof. Let $\phi \in \Gamma\left(\boldsymbol{\vee}\left(\llbracket \operatorname{Str} \rrbracket_{\mathcal{S}}^{\llbracket \operatorname{Str} \rrbracket_{\mathcal{S}}^{k}}\right)\right)=\Gamma\left(\llbracket \operatorname{Str} \rrbracket_{\mathcal{S}}^{\llbracket \operatorname{Str} \rrbracket_{\mathcal{S}}^{k}}\right)$. We have

$$
\lim (\Gamma(F)(\phi))=\lim \left(F_{\omega}(\phi)\right)=F_{\omega}(\phi)_{\omega}
$$

and

$$
\underline{F}(\lim (\phi))=\underline{F}\left(\phi_{\omega}\right)
$$

Now both of these are elements of $\llbracket \operatorname{Str} \rrbracket_{\text {Set }}^{\llbracket S t r} \rrbracket_{\text {Set }}^{k}$, meaning genuine functions in Set, so to show they are equal we use elements. Let $\vec{\sigma} \in \llbracket \operatorname{Str} \rrbracket_{\text {Set }}^{k}$.

We are then required to show

$$
\underline{F}\left(\phi_{\omega}\right)(\vec{\sigma})=F_{\omega}(\phi)_{\omega}(\vec{\sigma})
$$

Recall that $F=\Lambda\left(F^{\prime}\right)$ (exponential transpose) so $F_{\omega}(\phi)_{\omega}(\vec{\sigma})=F_{\omega}^{\prime}(\phi, \vec{\sigma})$. Now recall that composition in $\mathcal{S}$ is just composition of functions at each stage and products in $\mathcal{S}$ are defined pointwise and that next $_{\omega}$ is the identity function.

Moreover, the morphism hd gets mapped by $\Gamma$ to hd in Set and the same holds for tl. For the latter it is important that $\Gamma(\nabla(X))=\Gamma(X)$ for any $X$.

We thus get

$$
F_{\omega}^{\prime}(\phi, \vec{\sigma})=\operatorname{fold}_{\omega}\left(\left(\llbracket h_{f} \rrbracket_{\mathcal{S}}\right)_{\omega}(\mathbf{h d}(\vec{\sigma})),\left(\llbracket t_{f} \rrbracket_{\mathcal{S}}\right)_{\omega}(\phi, \mathbf{h d}(\vec{\sigma}), \vec{\sigma}, \mathbf{t l}(\vec{\sigma}))\right)
$$

And for $\underline{F}\left(\phi_{\omega}\right)(\vec{\sigma})$ we have

$$
\underline{F}\left(\phi_{\omega}\right)(\vec{\sigma})=\text { fold }_{\omega}\left(\llbracket h_{f} \rrbracket_{\text {Set }}(\mathbf{h d}(\vec{\sigma})),\left(\llbracket t_{f} \rrbracket_{\text {Set }}\right)\left(\phi_{\omega}, \mathbf{h d}(\vec{\sigma}), \vec{\sigma}, \operatorname{tl}(\vec{\sigma})\right)\right)
$$

It is now easy to see that these two are equal. The proof is by induction on the structure of $h_{f}$ and $t_{f}$. The variable cases are trivial, but crucially use the fact that next $_{\omega}$ is the identity. The cases for function symbols in $\Sigma_{A}$ are trivial since their denotations in Set are defined to be the correct ones. The case for $f$ goes through similarly since application at $\omega$ only uses $\phi$ at $\omega$. 
Theorem D.3. Let $\left(\Sigma_{1}, \Sigma_{2}\right)$ be a signature and $\mathcal{I}$ its interpretation. Let $\left(h_{f}, t_{f}\right)$ be a behavioural differential equation defining a $k$-ary function $f$ using function symbols in $\Sigma$. The right-hand sides of $h_{f}$ and $t_{f}$ define a term $\Phi_{f}^{\mathrm{g}}$ of type

$$
\Phi_{f}^{\mathrm{g}}: \triangleright(\underbrace{\mathrm{Str}^{\mathrm{g}} \rightarrow \mathrm{Str}^{\mathrm{g}} \rightarrow \cdots \mathrm{Str}^{\mathrm{g}}}_{k+1}) \rightarrow(\underbrace{\mathrm{Str}^{\mathrm{g}} \rightarrow \mathrm{Str}^{\mathrm{g}} \rightarrow \cdots \mathrm{Str}^{\mathrm{g}}}_{k+1}) .
$$

and a term $\Phi_{f}$ of type

$$
\Phi_{f}:(\underbrace{\operatorname{Str} \rightarrow \operatorname{Str} \rightarrow \cdots \operatorname{Str}}_{k+1}) \rightarrow(\underbrace{\operatorname{Str} \rightarrow \operatorname{Str} \rightarrow \cdots \operatorname{Str}}_{k+1}) .
$$

by using $\mathcal{L}_{a_{j}^{g}}\left(\mathcal{I}\left(g_{j}\right)\right)$ for interpretations of function symbols $g_{j}$.

Let $f^{\mathrm{g}}=$ fix $\Phi_{f}^{\mathrm{g}}$ be the fixed point of $\Phi_{f}^{\mathrm{g}}$. Then $f=\mathcal{L}_{k}\left(\right.$ box $\left.f^{\mathrm{g}}\right)$ is a fixed point of $\Phi_{f}$ which in turn implies that it satisfies equations $h_{f}$ and $t_{f}$.

Proof. Use Prop. D.2 together with Lemma D.1 together with the observation that Set is a full subcategory of $\mathcal{S}$ with $\Delta$ being the inclusion.

We also use the fact that for a closed term $u: A \rightarrow B$ (which is interpreted as a morphism from 1 to $\left.B^{A}\right)$ the denotation of $\mathcal{L}(u)$ at stage $\nu$ and argument $*$ is $\lim (\Gamma(u)(*))$.

\section{D.2 Discussion}

What we have shown is that for each behavioural differential equation that defines a function on streams and can be specified as a standalone function depending only on previously defined functions, i.e. it is not defined mutually with some other function, there is a fixed point. It is straightforward to extend to mutually recursive definitions by defining a product of functions in the same way as we did for a single function, but notationally this gets quite heavy.

More importantly, suppose we start by defining an operation $f$ on streams first, and the only function symbols in $\Sigma_{A}$ operate on $A$, i.e. all have type $A^{k} \rightarrow A$

for some $k$. Assume that these function symbols are given denotations in $\mathcal{S}$ as $\Delta(g)$ for some function $g$ in Set. Then the denotation in Set is just $g$.

The fixed point $f$ in $\mathcal{S}$ is then a morphism from 1 to the suitable exponential. Let $\bar{f}$ be the uncurrying of $f$. Then $\lim (\Gamma(f)(*))=\Gamma(\bar{f})$.

Thus if we continue defining new functions which use $f$, we then choose $\bar{f}$ as the denotation of the function symbol $f$. The property $\lim (\Gamma(u)(*))=\Gamma(\bar{f})$ then says that the $f$ that is used in the definition is the $f$ that was defined previously.

\section{E About Total and Inhabited Types}

An object in $\mathcal{S}$ is total and inhabited if all components are non-empty and all restriction functions are surjective. We have the following easy proposition. 
Proposition E.1. Let $P: \mathcal{S} \rightarrow \mathcal{S}$ be a functor such that if $X$ is a total and inhabited object, so is $P(X)$, i.e. $P$ restricts to the full subcategory of total and inhabited objects.

If $P$ is locally contractive then its fixed point is total and inhabited.

Proof. We use the equivalence between the full subcategory tiS of $\mathcal{S}$ of total and inhabited objects and the category of complete bisected non-empty ultrametric spaces $\mathcal{M}$. We know that the category $\mathcal{M}$ is an $M$-category and thus so is $t i \mathcal{S}$. It is easy to see that locally contractive functors in $\mathcal{S}$ are locally contractive in the $M$-category sense. Hence if $P$ is locally contractive and restricts to $t i \mathcal{S}$ its fixed point is in $t i \mathcal{S}$.

Corollary E.2. Let $P$ be a non-zero polynomial functor whose coefficients and exponents are total and inhabited. The functor $P \circ \triangleright$ is locally contractive and its unique fixed point is total and inhabited.

Proof. Products and non-empty coproducts of total and inhabited objects are total and inhabited. Similarly, if $X$ and $Y$ are total and inhabited, so is $X^{Y}$. So any non-zero polynomial functor $P$ whose coefficients are all total and inhabited restricts to $t i \mathcal{S}$. The functor restricts to $t i \mathcal{S}$ as well (but note that it does not restrict to the subcategory of total objects $t \mathcal{S}$ ). Polynomial functors on $\mathcal{S}$ are also strong and so the functor $P \circ$ is locally contractive. Hence by Prop. E.1 its unique fixed point is a total and inhabited object.

In particular guarded streams of any total inhabited type themselves form a total and inhabited type.

\footnotetext{
${ }^{2}$ Birkedal, L., Støvring, K., Thamsborg, J.: The category-theoretic solution of recursive metric-space equations. Theor. Comput. Sci. 411(47), 4102-4122 (2010)
} 